\title{
A pleiotropic chemoreceptor facilitates the functional coupling of pheromone production and perception
}

4 Cassondra Vernier ${ }^{1,2 \dagger}$, Kathleen M. Zelle ${ }^{1, \dagger}$, Nicole Leitner ${ }^{1}$, Xitong Liang ${ }^{3}$, Sean

5 Halloran ${ }^{4}$, Jocelyn G. Millar ${ }^{4}$, Yehuda Ben-Shahar ${ }^{1,5,{ }^{*}}$

$7 \quad{ }^{1}$ Department of Biology, Washington University in Saint Louis, 1 Brookings Drive, Saint

8 Louis, MO 63130, USA.

$9 \quad{ }^{2}$ Carl R. Woese Institute for Genomic Biology, University of Illinois, 1206 W. Gregory

10 Dr., Urbana, IL 61801, USA

11 '3epartment of Neuroscience, Washington University School of Medicine, 660 South

12 Euclid Avenue, St. Louis, MO 63110, USA

13 "Department of Entomology, University of California, Riverside, 900 University Avenue,

14 Riverside, CA 92521, USA

15 5ead Contact

16 tDenotes equal contribution

17 *Correspondence: benshahary@wustl.edu (Y.B.) 


\section{SUMMARY}

21 Optimal mating decisions depend on the robust coupling of signal production and

22 perception because independent changes in either could carry a fitness cost. However, 23 since the perception and production of mating signals are often mediated by different

24 tissues and cell types, the mechanisms that drive and maintain their coupling remain

25 unknown for most animal species. Here, we show that in Drosophila, sensory

26 perception and production of an inhibitory mating pheromone are co-regulated by Gr8a,

27 a member of the Gustatory receptor gene family. Specifically, we found that the

28 pleiotropic action of Gr8a independently regulates the perception of pheromones by the

29 chemosensory systems of males and females, as well as their production in the fat body

30 and oenocytes of males. These findings provide a relatively simple molecular

31 explanation for how pleiotropic receptors maintain robust mating signaling systems at

32 the population and species levels.

34 Keywords: Drosophila melanogaster; Vinegar fly; Fruit fly; Cuticular hydrocarbons;

35 Gr8a; Oenocyte. 


\section{INTRODUCTION}

The majority of sexually-reproducing animals use intricate mating signaling systems, which rely on a robust physiological coupling between the production and perception of species-specific signals since any independent changes in either the signal or the capacity to sense it would carry a fitness cost (Boake, 1991; Brooks et al., 2005; Hoy et al., 1977; Shaw et al., 2011; Shaw and Lesnick, 2009; Steiger et al., 2011; Sweigart, 2010; Symonds and Elgar, 2008; Wyatt, 2014). Previously published theoretical models have postulated that the maintenance of robust coupling between the production and perception of mating signals is driven by strong genetic linkage between the cellular and physiological processes that regulate mating-signal production and its perception, or alternatively, via the action of pleiotropic genes that control both processes (Boake, 1991; Butlin and Ritchie, 1989; Butlin and Trickett, 1997; Shaw et al., 2011; Shaw and Lesnick, 2009). Consequently, both mechanisms provide plausible explanations for how mating-signaling systems could remain stable and reliable at the population level while still retaining their capacity for future diversification, as necessitated for speciation (Chebib and Guillaume, 2021; Hoy et al., 1977; Kirkpatrick and Hall, 2004; Lande, 1980; Shaw et al., 2011; Shaw and Lesnick, 2009; Wiley et al., 2012).

Empirical data in support of the contribution of gene-linkage or pleiotropy to the maintenance of coupling between mating signal production and perception at the population level are rare (Chebib and Guillaume, 2021; Hoy et al., 1977; Shaw et al., 2011; Shaw and Lesnick, 2009; Wiley et al., 2012). Additionally, the complex characteristics of mating behaviors, and the species-specific signals that drive them, present a major barrier for identifying the actual molecular mechanisms and candidate pleiotropic genes that support the coupling between the production and perception of specific mating signals (Chenoweth and Blows, 2006; Singh and Shaw, 2012). How the functional coupling of the physiological processes responsible for the production and perception of mating signals remains robust is particularly puzzling since their perception is mediated by the peripheral sensory nervous system, while their production is restricted to specialized, non-neuronal pheromone producing cells (Chung and Carroll, 2015; Chung et al., 2014; McKinney et al., 2015). Notwithstanding, a previous Drosophila study has implied that the gene desat1, which encodes a fatty acid desaturase, directly contributes to both the perception and production of pheromones (Bousquet et al., 2012). However, subsequent studies have shown that desat1 expression is enriched in central neurons, and that the effect of desat1 mutations on the behavioral response to pheromones is not likely to be directly mediated via the modulation of pheromone perception by sensory neurons (Billeter et al., 2009). Furthermore, the effects of desat 1 mutations on the overall $\mathrm{CHC}$ profiles of both males and females are broad and lack specificity (Labeur et al., 2002). Together, these data suggest that desat 1 is not likely to act as a pleiotropic factor that directly couples the production and perception of mating pheromones in Drosophila. Consequently, the molecular identities of genes that may mediate the genetic and functional linkage between the production of insect mating pheromones by the coenocytes, and their perception by the chemosensory system, remained unknown.

79 Here we show that some pheromone-driven mating behaviors in Drosophila depend on 80 the pleiotropic action of Gr8a, a member of the Gustatory receptor gene family (Lee et 
al., 2012; Shim et al., 2015), which contributes to both the perception of inhibitory mating signals in pheromone-sensing neurons, and independently, to the production of inhibitory mating pheromones in non-neuronal abdominal pheromone-producing oenocytes. Together, these data provide a relatively simple molecular explanation for how genetic linkage could maintain functional coupling between the independent cellular and physiological processes that drive pheromone perception and production.

\section{RESULTS}

\section{Some gustatory-like receptors exhibit enriched expression in abdominal tissues}

Similar to other insect species, Drosophila cuticular hydrocarbons (CHCs), or long-chain fatty acids synthesized by the fat body and oenocytes (Billeter et al., 2009; Gutierrez et al., 2007), provide a hydrophobic desiccation barrier, as well as play an important role as pheromones in regulating diverse behaviors, including mating (Blomquist and Bagnères, 2010; Chung and Carroll, 2015; Ferveur, 2005; McKinney et al., 2015). Specifically, complex blends of $\mathrm{CHCs}$ are often utilized by insects to communicate sex identity and female mating status, as well as to define the behavioral reproductive boundaries between closely related species (Ben-Shahar, 2015; Billeter et al., 2009; Chung and Carroll, 2015; Chung et al., 2014; Dweck et al., 2015; Lu et al., 2012; Lu et al., 2014; Yew and Chung, 2015).

While some of the genes and pathways that contribute to $\mathrm{CHC}$ synthesis in Drosophila are known (Blomquist and Bagnères, 2010; Chung et al., 2014; Ferveur, 2005; Howard

102 and Blomquist, 2005; McKinney et al., 2015), the molecular identities of most CHC receptors remain unknown. Current models stipulate that the perception of volatile

104

105 $\mathrm{CHCs}$ is mediated by olfactory sensory neurons (ORNs) located in the antennae and maxillary palps, while less volatile $\mathrm{CHCs}$ are sensed by specialized gustatory-like receptor neurons (GRNs) in the appendages (legs and wings), female genitalia, and the proboscis (Benton et al., 2007; Clowney et al., 2015; Datta et al., 2008; Koh et al., 2014; Kurtovic et al., 2007; Lebreton et al., 2014; Lu et al., 2012; Lu et al., 2014; Pikielny, 2012; Thistle et al., 2012; Toda et al., 2012; van der Goes van Naters and Carlson, 2007; Vijayan et al., 2014).

111 Consequently, we chose to examine members of the Gustatory receptor (Gr) gene

112 family as candidate pleiotropic genes that might contribute to both the perception and production of pheromonal mating signals in Drosophila. Because several family members have already been implicated in the detection of specific excitatory and inhibitory pheromones (Bray and Amrein, 2003; Miyamoto and Amrein, 2008; Moon et al., 2009; Watanabe et al., 2011), and the majority of genes that encode family members are already known to be enriched in GRNs (Clyne et al., 2000; Dunipace et al., 2001; Scott et al., 2001; Wang et al., 2004), we reasoned that any pleiotropic $\mathrm{Gr}$ genes should be also expressed in the abdominal oenocytes (Billeter et al., 2009). We tested this by using an RT-PCR screen, which revealed that 24 out of the 60 members of the Gr family are expressed in abdominal tissues of adult Drosophila (Table 1). This

122

123 suggests that at least some Grgenes may contribute to both the perception and production of mating signals in Drosophila. 


\section{Gr8a is a chemosensory receptor with sexually dimorphic expression in} abdominal cells

Although several members of the Gr gene family, including Gr68a, Gr32a, Gr66a, Gr39a, and Gr33a, were previously linked to the sensory perception of mating pheromones (Bray and Amrein, 2003; Lacaille et al., 2007; Miyamoto and Amrein, 2008; Moon et al., 2009; Watanabe et al., 2011), none of these candidate genes were identified in our initial RT-PCR screen for Gr genes expressed in abdominal tissues of either males or females (Table 1). However, Gr8a, which was indicated by our screen as being a male-specific abdomen-enriched receptor (Table 1) (Park and Kwon, 2011), was previously shown to play a role in the chemosensation of the non-proteinogenic amino acid L-Canavanine (Lee et al., 2012; Shim et al., 2015). Because our initial expression screen was based on whole-abdomen RNAs, we next used a GAL4 transgenic driver to determine which abdominal cells express Gr8a. We found that, as was previously reported (Lee et al., 2012), Gr8a is expressed in 14-16 GRNs in the proboscis (Figure 1A-B), as well as in two paired GRNs in the pretarsus of the prothoracic legs in males (Figure 1C) and females (Figure 1D). We also observed Gr8a expression in abdominal oenocyte-like cells in males (Figure 1E) but not females (Figure 1F). The male-biased expression in the abdomen was further supported by qRT-PCR analysis (Figure $1 \mathrm{G}$ ). These data further indicate that in addition to its chemosensory functions, Gr8a may also contribute to oenocyte physiology.

To further examine the spatial expression of Gr8a in males, we used a membrane bound GFP reporter to trace the axonal projection patterns of Gr8a-expressing GRNs in the prothoracic legs. We found that in contrast to the primary, sexually dimorphic ppk23expressing pheromone-sensing GRNs (Lu et al., 2012; Lu et al., 2014; Thistle et al., 2012; Toda et al., 2012), the axons of tarsal Gr8a-expressing neurons ascend to the brain and do not cross the midline of the ventral nerve cord (VNC) in males (Figure $1 \mathrm{H}$ ). Likewise, we found that Gr8a-expressing GRNs do not co-express the sex determination factor fru (Figure 1I) or the ion channel ppk23 (Figure 1J), which are were previously assumed to be expressed in all pheromone-sensing GRNs in the fly appendages. These data indicate that Gr8a-expressing GRNs in the prothoracic tarsal segments possibly represent a distinct subclass of pheromone-sensing GRNs.

In the male abdomen, we found that Gr8a is co-expressed with the oenocyte specific desat1 driver (Billeter et al., 2009), as well as possibly in desat1-negative fat-body-like cells (Figure 1K-M). To better understand how Gr8a might function in non-neuronal oenocytes, we next characterized the subcellular localization of the native Gr8a protein in abdominal tissues, by using CRISPR/Cas9 genome editing to generate an endogenous GFP-tagged allele of Gr8a. Subsequently, immunohistochemical staining of abdominal tissues from Gr8a-GFP males with an anti-GFP antibody revealed that the receptor protein is enriched in vacuolar membranes in some oenocyte clusters (Figure $1 \mathrm{~N})$. Together, these data indicate that in addition to its possible role in the perception of

166 mating pheromones in the male. 


\section{Gr8a activity contributes to mating decisions in females}

We next hypothesized that if Gr8a is a pleiotropic gene that independently contributes to the production of a mating pheromone in males, and its chemosensory perception in females, then the knockdown of Gr8a in either males or females should have similar effects on female mating behavior. Therefore, we first investigated whether Gr8a, and the GRNs that express it, are required for sensory functions associated with female mate choice by using single-pair courtship assays (Lu et al., 2012; Lu et al., 2014). We found that blocking neuronal transmission in female Gr8a-expressing GRNs by the transgenic expression of tetanus toxin (TNT) shortens copulation latency relative to wild-type females, when courted by wild-type males (Figure 2A). Similarly, homozygous (Figure 2B) and hemizygous (Figure 2C) Gr8a-null females exhibited shorter copulation latencies when courted by wild type males, which can be rescued by driving the expression of the Gr8a cDNA by Gr8a-GAL4 (Figure 2D). In contrast, genetic manipulations of Gr8a did not affect male courtship behavior as measured by courtship latency and index towards wild-type females (Supplemental Figure 1). These data suggest that $\mathrm{Gr} 8 \mathrm{a}$ is required for regulating female mating receptivity via the chemosensory detection of male-borne inhibitory mating pheromones.

Because Gr8a expression is specifically enriched in male oenocytes (Figure 1K-M), we next tested the hypothesis that Gr8a also plays a role in the production and/or release of inhibitory mating signals by males. We found that wild-type virgin females exhibited shorter copulation latencies towards Gr8a mutant males relative to wild-type controls (Figure 2E). These data indicate that the Gr8a mutant males produce and/or release lower levels of inhibitory mating pheromones relative to wild type controls. Together, these behavioral studies suggest that $\mathrm{Gr} B a$ is a pleiotropic gene that regulates both the production of an inhibitory mating signal in the male oenocytes, and its perception by the chemosensory system in females.

\section{Gr8a regulates the copulatory transfer, and the post-mating perception, of inhibitory pheromones by males}

Mating decisions in $D$. melanogaster rely on a balance between excitatory and inhibitory drives (Billeter et al., 2009; Clowney et al., 2015; Kallman et al., 2015; Krupp et al., 2008; Laturney and Billeter, 2016). Therefore, male-borne inhibitory signals may help females optimize mate choices by delaying their decision to copulate with specific males. Additionally, previous studies showed that, in order to increase their fitness, Drosophila males transfer inhibitory mating pheromones to females during copulation, which subsequently lowers the overall attractiveness of mated females to other males (Averhoff and Richardson, 1974; Datta et al., 2008; Jin et al., 2008; Kurtovic et al., 2007; Miyamoto and Amrein, 2008; Yang et al., 2009). We found that Gr8a mutant males were more likely to court mated females than wild-type controls (Figure $2 \mathrm{~F}$ ), suggesting that Gr8a is also required in males for the sensory recognition of the inhibitory signals that label the post-mating status of females. We also found that wildtype males failed to recognize the mating status of wild-type females that were previously mated with Gr8a mutant males (Figure 2F). These data indicate that Gr8a is also important for the production of inhibitory pheromones that are transferred from 
males to females during copulation. Together, these findings suggest that Gr8a is responsible for the production and perception of transferrable inhibitory mating signals that advertise post-mating status in females. The simplest overall interpretation of these data is that Gr8a is a pleiotropic factor, which independently contributes to the production/ transfer of male inhibitory mating pheromones, as well as their sensory perception in both males and females.

\section{Gr8a contributes to quantitative and qualitative attributes of the pheromone profiles of males and mated females}

Because our data indicate that the Gr8a mutation has a dramatic effect on the copulation latency of mated females and the ability of males to detect the mating status of females, we hypothesized that Gr8a is contributing to the production and/or transfer of an inhibitory pheromone in males. Therefore, we next examined whether the Gr8a mutation has a direct effect on qualitative and quantitative aspects of male and matedfemale $\mathrm{CHC}$ profiles. We found that the overall $\mathrm{CHC}$ profile of Gr8a mutant males is both qualitatively (Figure $3 \mathrm{~A}$ ) and quantitatively different from that of wild-type males (Figure 3B-C and Table 2). In particular, the Gr8a mutation affects the levels of several alkenes and methyl-branched alkanes, which have been implicated in mate choice behaviors in diverse Drosophila species (Billeter et al., 2009; Billeter and Levine, 2013; Chung and Carroll, 2015; Chung et al., 2014; Dyer et al., 2014; Shirangi et al., 2009).

Although the exact mechanism by which Gr8a might be regulating the levels of specific $\mathrm{CHCs}$ remains unknown, we found that the expression levels of the desaturases desat 1 and CG8630, which play a role in the biosynthesis of alkenes (Chung and Carroll, 2015), are affected by the Gr8a mutation in the male abdomen (Figure 3D). We also found that the overall qualitative aspects of the $\mathrm{CHC}$ profiles of wild-type females were not affected by mating with either Gr8a mutant or wild-type males (Figure 3E). However, quantitative analyses of individual $\mathrm{CHCs}$ revealed that nonacosane $\left(\mathrm{C}_{29}\right)$ is higher in females that mated with Gr8a mutant males relative to those that mated with wild-type males (Figure 3F). Together, these data suggest that Gr8a action in oenocytes contributes to the production of some cuticular alkenes and methyl-branched alkanes in males, which possibly function as inhibitory mating pheromones.

Since the Gr8a mutation is not spatially restricted in Gr8a mutant males, it is possible that at least some of the effects of the Gr8a mutation on the pheromone profiles of males are indirectly mediated via its action in pheromone-sensing GRNs, instead of directly mediated via its action in oenocytes. Therefore, we next examined the effect of oenocyte-specific Gr8a knockdown on the production of male CHCs. We found that oenocyte-specific Gr8a RNAi knockdown in males leads to significant changes in their overall CHC profile relative to control males (Figure 3G). In contrast, fat-body-specific knockdown of Gr8a has no effect on the $\mathrm{CHC}$ profiles of males (Figure $3 \mathrm{H}$ ). These data suggest that Gr8a is likely to play an oenocyte-specific role in the production of male CHCs. Together, our behavioral and pheromonal data indicate that Gr8a action contributes to mating decisions in females by co-regulating the perception of an inhibitory mating pheromone by females and males, as well as its production by males. This is consistent with a pleiotropic function for Gr8a. 


\section{Gr8a-associated CHCs inhibit normal courtship behaviors}

To further characterize whether any of the individual CHCs regulated by Gr8a actually function as inhibitory mating pheromones, we tested the effect of perfuming naïve males with individual candidate $\mathrm{CHCs}$ on the copulation latency of wild-type females (BenShahar et al., 2010; Ben-Shahar et al., 2007; Leitner and Ben-Shahar, 2020; Lu et al., 2012; Lu et al., 2014). We found that wild-type females did not copulate with Gr8a mutant males that were perfumed with the alkenes $9-C_{25}, 7-C_{25}$, and $7-C_{27}$ (Figure 4A). Similarly, we found that wild-type males exhibited a longer courtship latency and lower courtship index towards wild-type females perfumed with 9- $\mathrm{C}_{25}$ (Figure 4B-D), and exhibited longer copulation latency towards wild-type females perfumed with $7-\mathrm{C}_{25}$ (Figure 4E-G). In contrast, perfuming wild-type females with 7- $\mathrm{C}_{27}$ had no effect on male courtship or female mating latency (Figure $4 \mathrm{H}-\mathrm{J}$ ). These data suggest that at least some of the $\mathrm{CHCs}$ regulated by Gr8a activity in the male oenocytes are inhibitory mating pheromones.

\section{Variations in Gr8a contribute to species-specific male pheromonal profiles across the Drosophila genus}

As populations diversify, pheromonal signals and their receptors often have to co-evolve to maintain behavioral species boundaries (Boake, 1991; Khallaf et al., 2021; Symonds and Elgar, 2008; Symonds and Wertheim, 2005). One possible mechanism for maintaining the functional coupling of coevolving signal-receptor pairs during speciation is pleiotropy (Boake, 1991; Shaw et al., 2011; Singh and Shaw, 2012). Because our data suggest that $\mathrm{Gr} 8 \mathrm{a}$ is a pleiotropic pheromone receptor, we tested the hypothesis that cross-species variations in the Gr8a coding sequence may have contributed to the rapid evolution of mating pheromones in the Drosophila species group (Khallaf et al., 2021; Shahandeh et al., 2018; Shirangi et al., 2009). To test this hypothesis, we first performed a phylogenetic analysis of Gr8a orthologs across Drosophila species, which indicated that Gr8a is a conserved, sexually dimorphic receptor across the Drosophila genus (Figure 5A-B). Furthermore, alignment of Gr8a proteins across all the major Drosophila clades revealed that, in spite of its high overall sequence conservation, the Gr8a receptor has at least one phylogenetically variable domain (magenta frame, Figure 5C), which includes the second intracellular and extracellular domains (Figure 5D).

Although the ligand-binding domains of the insect Gr gene family have not been identified yet, these data suggest that this phylogenetically variable protein domain in Gr8a may contribute to species-specific shifts in ligand-binding specificity and/ or sensitivity across the Drosophila genus. Therefore, we next tested whether the transgenic rescue of the Gr8a null allele via ectopic expression of Gr8a cDNAs from different Drosophila species is sufficient to drive changes in the $\mathrm{CHC}$ profile of $D$. melanogaster males. By using a cross-species male mate-choice assay, we found that while $D$. melanogaster males are generally promiscuous, they do court $D$. mojavensis females at a significantly lower proportion than conspecific females. Because these assays are performed under red light, which eliminates visual mating cues, these data suggested that the lower sex drive towards $D$. mojavensis females is pheromone- 
dependent (Figure 5E). Subsequently, we generated transgenic $D$. melanogaster lines which express either the $D$. mojavensis or the $D$. melanogaster Gr8a cDNAs driven by an oenocyte-specific GAL4 in the background of the Gr8a null allele. Comparison of male $\mathrm{CHC}$ profiles across the two genotypes revealed that rescuing the Gr8a mutation by Gr8a cDNAs from these two distantly related species resulted in significantly different male $\mathrm{CHC}$ profiles (Figure 5F). These data indicate that species-specific Gr8a coding variations are sufficient to drive differential $\mathrm{CHC}$ production by the male oenocytes, and suggest that pleiotropic pheromone receptors may have played a role in driving the rapidly evolving behavioral mating boundaries in Drosophila.

\section{DISCUSSION}

The data presented here demonstrate that Gr8a is a pleiotropic chemoreceptor that coregulates the perception and production of an inhibitory pheromonal signal that plays an important role in mating behaviors of both $D$. melanogaster sexes. How Gr8a, a member of a canonical chemoreceptor family, might also contribute to the production of pheromonal signals is not obvious. In some better understood secretory cell types, autoreceptors are essential for the regulation of synthesis and secretion rates. For example, dopaminergic and serotonergic cells regulate rates of synthesis and release of their respective neuromodulators by the action of autoreceptors, which act via signaling feedback in response to changes in the extracellular concentrations of the secreted molecule (Ford, 2014; Stagkourakis et al., 2016). Therefore, one possible explanation for how Gr8a might regulate the synthesis and/or secretion of specific $\mathrm{CHCs}$ is by acting as an oenocyte-intrinsic autoreceptor, which regulates the synthesis of specific $\mathrm{CHCs}$ by providing feedback information about their levels in internal stores and/ or extracellularly (Figure 6).

Recent studies have indicated that Drosophila bitter receptor neurons typically express multiple $\mathrm{Gr}$ genes, and that bitter receptor ligand specificity is determined via combinatorial heteromeric receptor complexes (Dweck and Carlson, 2020; Shim et al., 2015; Sung et al., 2017). Gr8a is specifically required for the sensory perception of the feeding deterrent L-canavanine (Lee et al., 2012; Shim et al., 2015), but not for the detection of other bitter feeding deterrents such as caffeine, strychnine, and umbelliferone (Lee et al., 2009; Poudel et al., 2015). Our data indicate that similar to other Drosophila "bitter" taste receptors (Lacaille et al., 2007; Moon et al., 2009), Gr8a contributes to inhibitory sensory inputs in the contexts of both feeding and mating decisions. In the context of feeding, Gr8a-dependent perception of L-canavanine is mediated via its heterotrimeric interaction with Gr66a and Gr98b in bitter sensing neurons in the proboscis (Shim et al., 2015). However, although both Gr66a and Gr98b were also identified in our initial screen for receptors enriched in the adult abdomen, we found that Gr66a is expressed in both sexes and Gr98b is specifically enriched in females (Table 1). Therefore, we conclude that Gr8a-dependent contributions to sensory functions associated with mating decisions are independently driven via its heteromerization with different Gr genes than those that drive feeding-specific decisions. 
Although we do not yet know the specific chemical identity of the ligand of Gr8a, previous studies indicated that at least two inhibitory mating pheromones, 11-cisvaccenyl acetate (cVA) and $\mathrm{CH} 503$, are transferred from males to females during copulation. While our data suggest that the Gr8a mutation affects the level of cVA expressed by males, it is unlikely that either cVA or $\mathrm{CH} 503$ are the putative $\mathrm{Gr} 8 \mathrm{a}$ ligands because the volatile cVA acts primarily via the olfactory receptor Or67d (Benton et al., 2007; Datta et al., 2008; Kurtovic et al., 2007), and CH503 has been reported to signal via Gr68a-expressing neurons, which are anatomically distinct from the $\mathrm{Gr} 8 \mathrm{a}$ GRNs we describe here (Figure 1A-B) (Shankar et al., 2015; Yew et al., 2009). Instead, our analyses of the effect of the Gr8a mutation on the $\mathrm{CHC}$ profile (Figure 3), and our results of the perfuming behavioral studies (Figure 4), suggest that the alkenes $5-\mathrm{C}_{25}, 7$ $\mathrm{C}_{25}$, and $7-\mathrm{C}_{27}$, which seem to act as inhibitory mating signals as well, are potentially the ligands of Gr8a.

Overall, our studies indicate that pleiotropic receptors, such as Gr8a, contribute to the physiological coupling between the production and perception of some mating pheromones by acting as both a sensory receptor in pheromone-sensing neurons, and possibly as an autorecepor for the same chemical in the pheromone-producing oenocytes. Our finding that Gr8a is also a sexually dimorphic receptor that is conserved across the Drosophila genus, with at least one phylogenetically variable domain (Figure $5 A-C)$, suggests that it might also drive the divergence of mating signaling systems in association with rapid speciation. This is supported by our finding that rescuing the Gr8a mutation in $D$. melanogaster with a Gr8a cDNA from a distant species, $D$. mojavensis, leads to the development of a male $\mathrm{CHC}$ profile that is different from the profile of mutant males rescued with the D. melanogaster Gr8a cDNA (Figure 5F).

Studies in other animal species suggest that receptor pleiotropy likely plays a role in mating signaling via other sensory modalities including auditory communication in crickets (Heinen-Kay et al., 2020; Hoy et al., 1977; Wiley et al., 2012) and visual communication in fish (Fukamachi et al., 2009). While the specific genes and signaling pathways that mediate the coupling of the mating signals and their receptors in these mating systems remain mostly unknown, these data suggest that genetic linkage in signal-receptor pairs important for mating communication is likely to be more common than previously thought. Therefore, the genetic tractability of $D$. melanogaster, in combination with the diversity of mating communication systems in this species-rich phylogenetic group, provide a unique opportunity for understanding the evolution and mechanisms that drive and maintain the robustness of mating systems at the genetic, molecular, and cellular levels.

\section{ACKNOWLEDGMENTS}

We thank members of the Ben-Shahar lab for comments on earlier versions of the manuscript. We thank Joshua Krupp (University of Toronto) for assistance with perfuming studies, Nabeel Chowdhury and Deanna Simon for assistance with qRT-PCR analysis, and Paula Kiefel for technical help with generating transgenic flies. This work was supported by NSF grants 1322783, 1754264, and 1707221, and NIH grant

386 NS089834 awarded to Y. B-S. Stocks obtained from the Bloomington Drosophila Stock 
387 Center (NIH P40OD018537) were used in this study. Wild-type Drosophila species were 388 obtained from the National Drosophila Species Stock Center at Cornell University.

389

390 AUTHOR CONTRIBUTIONS

391 K.M.Z., C.V., J.G.M. and Y.B-S designed experiments. K.M.Z., C.V., N.L., X.L., S.H., 392 J.G.M. and Y.B-S collected and analyzed data. K.M.Z., C.V., N.L. and Y.B-S wrote the 393 manuscript.

394

395 DECLARATION OF INTERESTS

396 The authors declare no competing interests. 

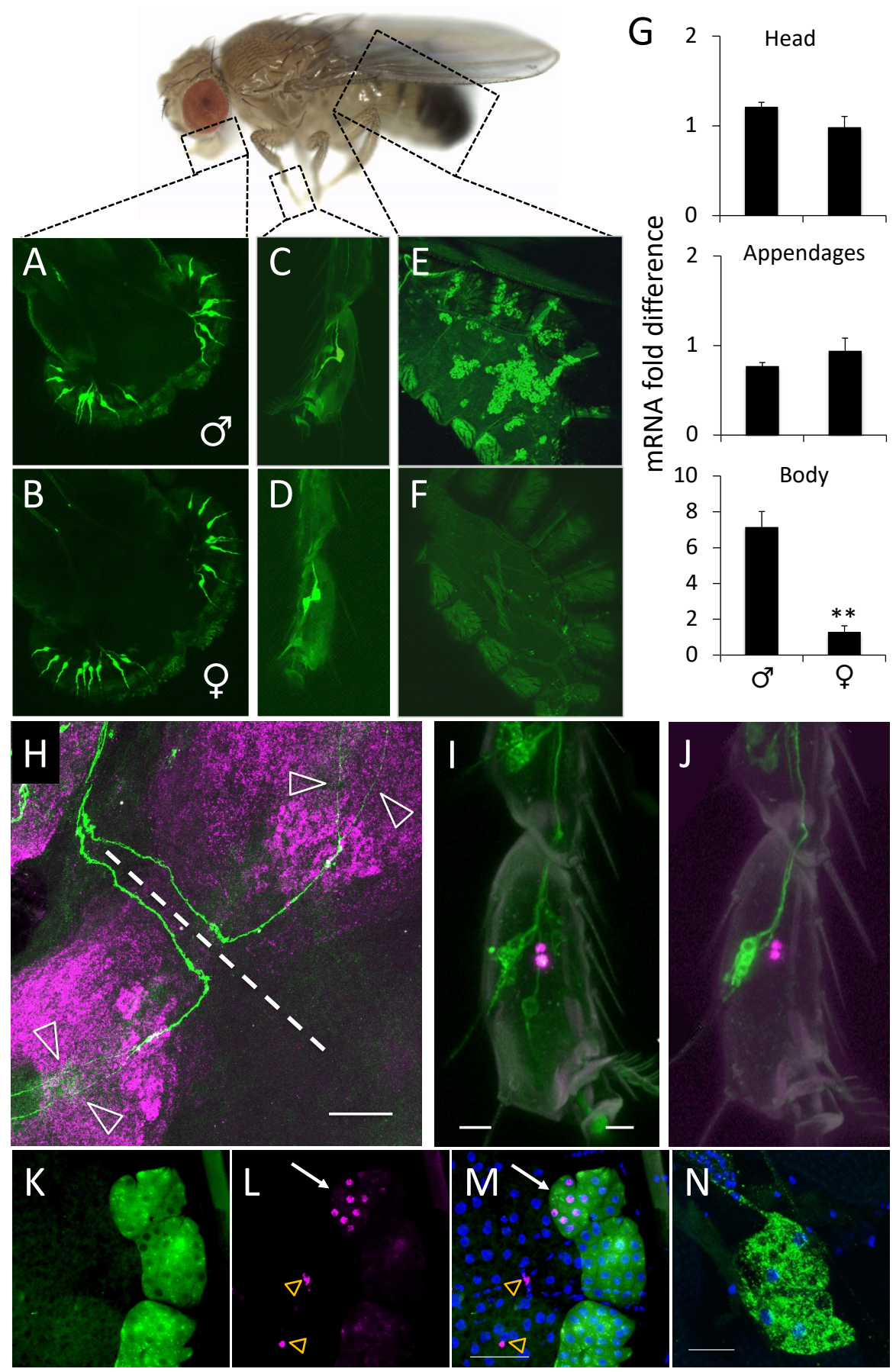

399 Figure 1. Gr8a is a sexually dimorphic chemosensory receptor. (A-F) Gr8a is 400 expressed in the proboscis (A-B) and prothoracic legs (C-D) of both males (top) and 401 females (bottom), but is only expressed in the abdomen of males (E-F). Cells labeled by 402 Gr8a-GAL4. (G) Gr8a has sexually dimorphic mRNA expression in the bodies of flies. 
403 Relative mRNA levels were measured by real-time quantitative RT-PCR. **,$p<0.01$ 404 Mann Whitney Rank Sum Test, n=3/group. (H-J) Gr8a-expressing GRNs represent a 405 distinct subclass of pheromone sensing neurons. $(\mathrm{H})$ Axonal projection patterns in the 406 T1 VNC neuromere in a Gr8a-GAL4>UAS-CD8::GFP male (green). Arrowheads, 407 individual axons; dashed line, midline of the VNC. Magenta, neuropil marker (nc82). (I) 408 Confocal z-stack of a male fruP1-LexA>LexAop-myrGFP (green); Gr8a-GAL4>UAS409 Red-Stinger (magenta) prothoracic leg. (J) Confocal z-stack of a male ppk23410 LexA>LexAop-CD8::GFP (green); Gr8a-GAL4>UAS-Red-Stinger (magenta) prothoracic 411 leg. (K-M) Gr8a is expressed in oenocytes and other abdominal cells. Confocal z-stack 412 images of oenocytes in a Gr8a-GAL4>UAS-CD8::GFP; desat1>luciferase male: (K) 413 desat1 (green); (L) Gr8a (magenta); (M) Merge. Blue, DAPI. White arrow, expression of 414 Gr8a in oenocytes; yellow arrows, expression of Gr8a in other abdominal cells. (N) 415 GR8A protein is enriched in abdominal cells. Confocal z-stack of a GFP-tagged Gr8a 416 allele in male abdominal cells; green, anti-GFP; blue, DAPI. Scale bars $=50 \mu \mathrm{m}$. 

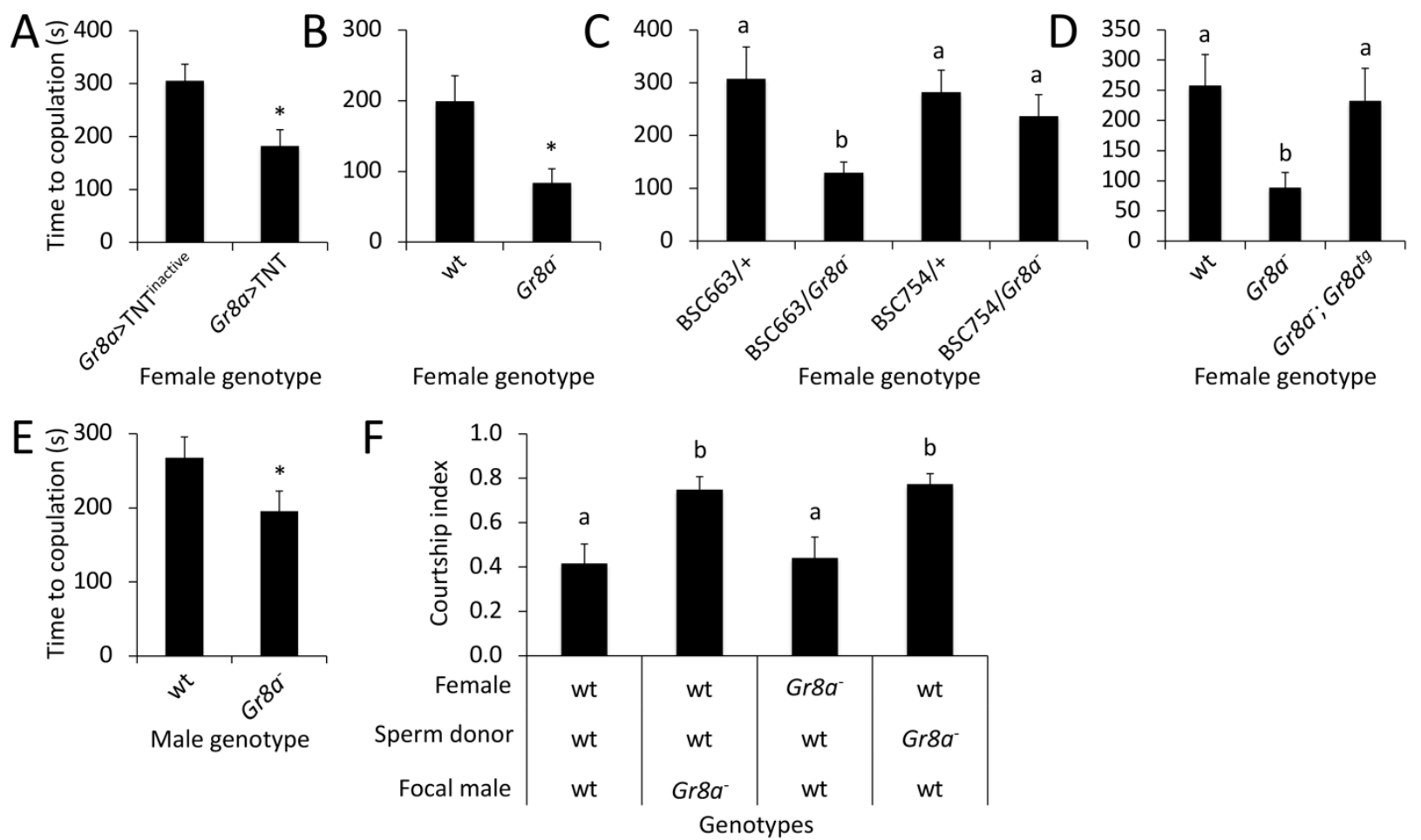

Figure 2. Gr8a activity contributes to the perception and production of an inhibitory signal associated with mating decisions in males and females. (A) Blocking neural activity in female Gr8a-expressing sensory neurons (Gr8a>TNT) shortens copulation latency relative to wild-type controls (Gr8a>TNTinactive). (B-C) Homozygous (B) or hemizygous (C) Gr8a null females show shortened copulation latency relative to wild-type controls. $\mathrm{Df}(1) \mathrm{BSC} 663$ is a deficiency that covers the Gr8a locus. Df(1)BSC754 was used as a control. (D) Expression of Gr8a cDNA with the Gr8a promoter (Gr8a-;Gr8a ${ }^{\text {tg }}$ ) rescues the copulation latency phenotype in Gr8a mutant females. (E) Wild-type females exhibit shortened copulation latency when courted by Gr8a mutant males relative to wild-type males. (F) Gr8a mutant males do not recognize the mating status of females, and have a reduced transfer of inhibitory mating pheromones during copulations. Female, female genotype; Sperm donor, genotype of males mated first with focal females; Focal male, genotypes of experimental males presented with mated females. Different letters above bars indicate statistically significant Tukey's HSD post hoc contrasts between groups. Panels C, D, and F: $p<0.05$ ANOVA, $n>15 / g r o u p$. Panels A, B, E: *, $p<0.05$, Mann Whitney Rank Sum Test, $n>15 / g r o u p$. All assays performed under red light conditions. 

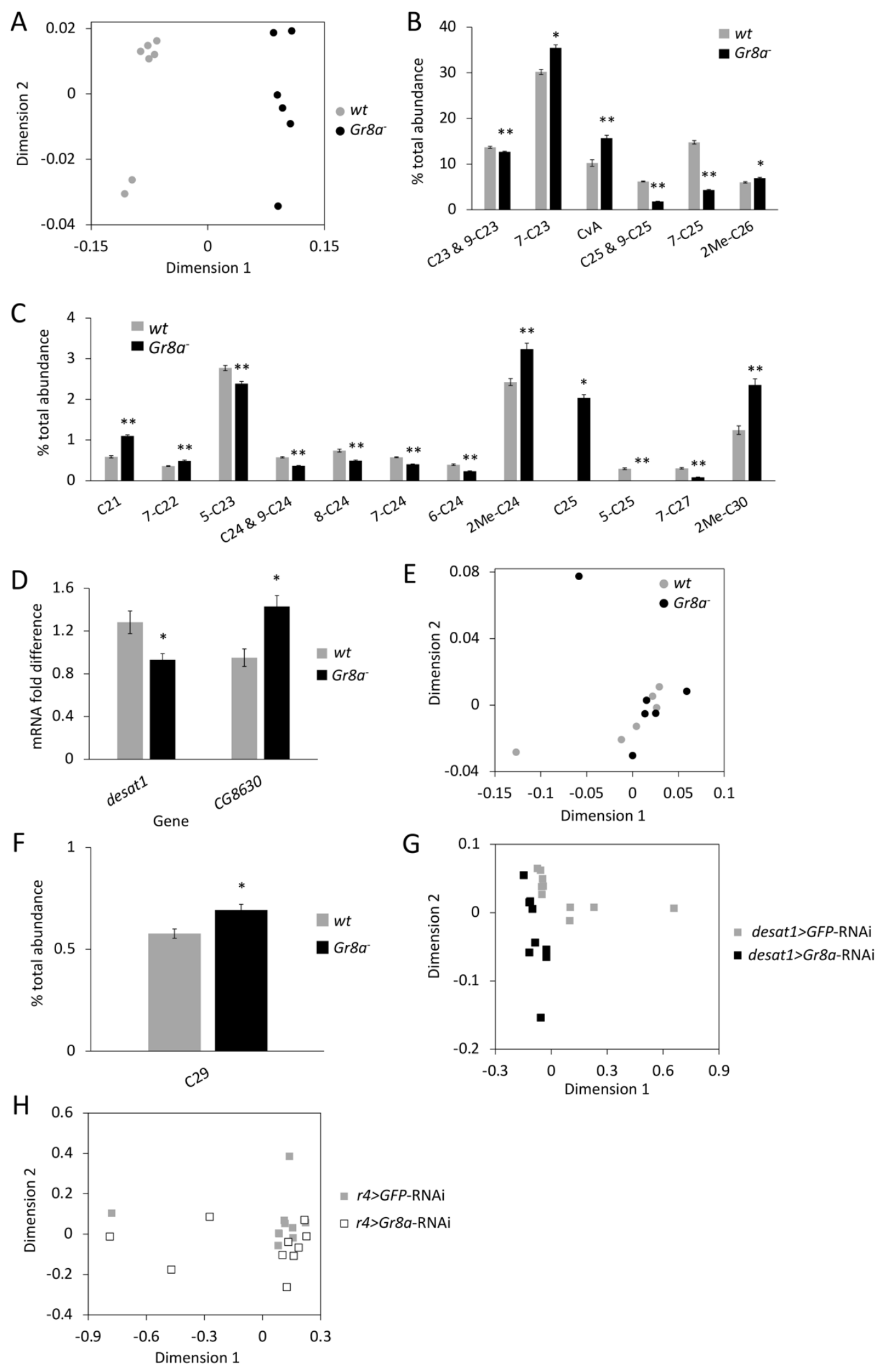
437 Figure 3. The Gr8a mutation affects the pheromone profiles of males and mated

438 females. (A) Wild-type (wt) and Gr8a mutant (Gr8a') males differ in $\mathrm{CHC}$ profile.

$439 \mathrm{p}<0.001$, Permutation MANOVA. (B-C) The Gr8a mutation affects the levels of

440 individual $\mathrm{CHCs}$ in males. (B) $\mathrm{CHCs}$ found at high proportions in males. (C) $\mathrm{CHCs}$ found

441 at low proportions in males. Only affected CHCs are shown. See Table 2 for the

442 complete list. *, $p<0.05,{ }^{* *}, p<0.001$, Student's t-test or Mann Whitney Rank Sum Test,

$443 \mathrm{n}=6$ (Gr8a-) or 7 (wt). (D) The Gr8a mutation affects the expression level of several

444 desaturase genes. Only affected genes are shown. See Table 3 for the complete list. *,

$445 \mathrm{p}<0.05$, Student's t-test, $\mathrm{n}=4$ /group. (E) Females mated with wild-type or Gr8a mutant

446 males do not differ in $\mathrm{CHC}$ profile. $p=0.570$, Permutation MANOVA. (F) Nonacosane

$447 \quad\left(\mathrm{C}_{29}\right)$ differs between females mated with wild-type and Gr8a mutant males. See Table 4

448 for complete list of mated-female CHCs. *, $p<0.05$, Student's t-test, $n=6 / g r o u p$. (G)

449 Control (desat1 > GFP-RNAi) and oenocyte-specific Gr8a knockdown (desat1 > Gr8a-

450 RNAi) males differ in $\mathrm{CHC}$ profile. $\mathrm{p}<0.001$, Permutation MANOVA. (H) Control $(r 4>$

451 GFP-RNAi) and fat body-specific Gr8a knockdown ( $r 4>\mathrm{Gr} 8 \mathrm{a}-\mathrm{RNAi}$ ) males do not differ

452 in CHC profile. $p=0.298$, Permutation MANOVA. Panels A, E, G, and H depicted as

453 Nonmetric Multidimensional Scaling (NMDS) plots with Bray-Curtis dissimilarity. 

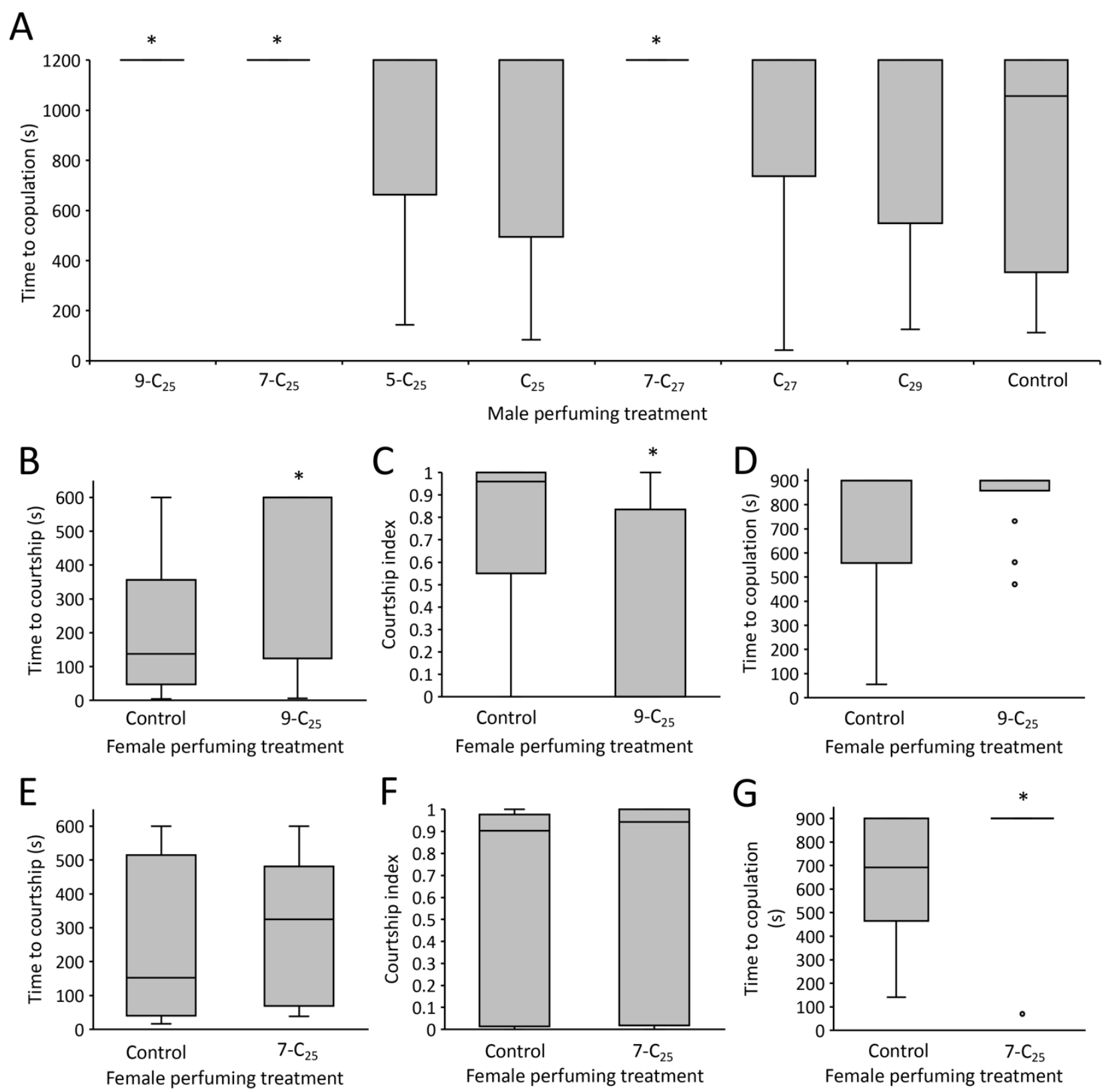

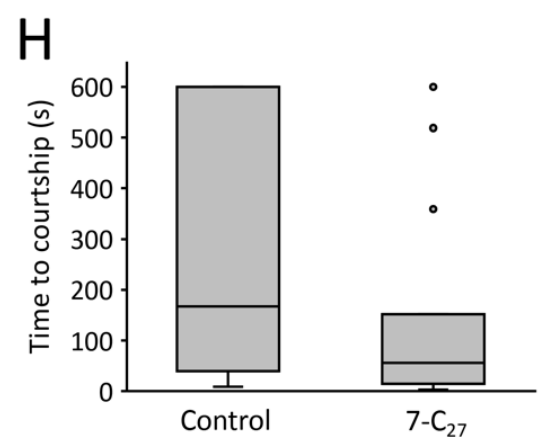

Female perfuming treatment

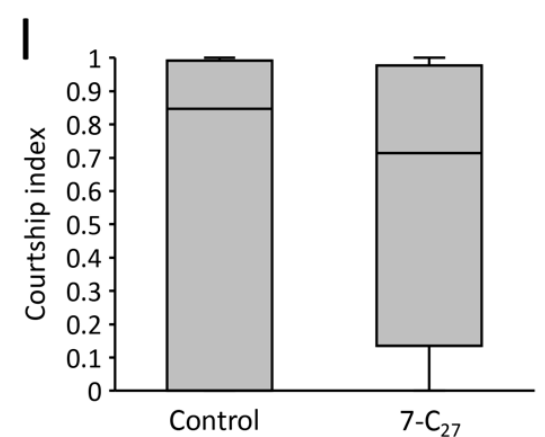

Female perfuming treatment

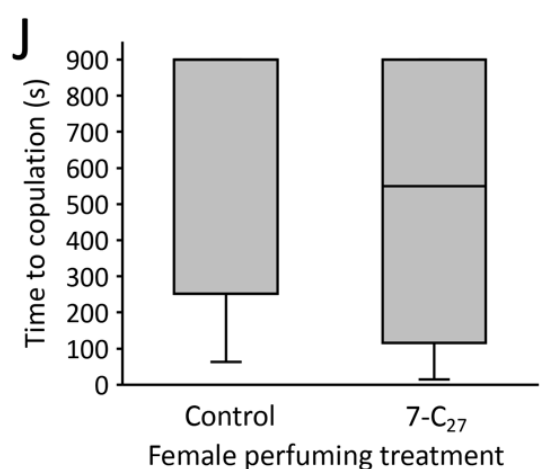


456 Figure 4. Gr8a-associated alkenes inhibit normal courtship behaviors. (A)

457 Perfuming males with exaggerated amounts of several alkenes increases copulation 458 latency compared to control males. (B-D) Perfuming females with $9-C_{25}$ increases 459 courtship latency (B), decreases courtship index (C), but does not affect copulation 460 latency (D) compared to control females. (E-G) Perfuming females with 7- $\mathrm{C}_{25}$ does not 461 affect courtship latency $(E)$ or index $(F)$, but increases copulation latency $(G)$ compared 462 to control females. (H-J) Perfuming females with 7- $\mathrm{C}_{27}$ does not affect courtship latency $463(\mathrm{H})$, courtship index $(\mathrm{I})$, or copulation latency $(\mathrm{J})$ compared to control females. Asterisks 464 above bars indicate statistically significant contrasts compared to control flies, $p<0.05$, 465 Kruskal-Wallis Test followed by Dunn's Test (A) or Mann Whitney Rank Sum Test (B-J), $466 \mathrm{n}=15 /$ group. 


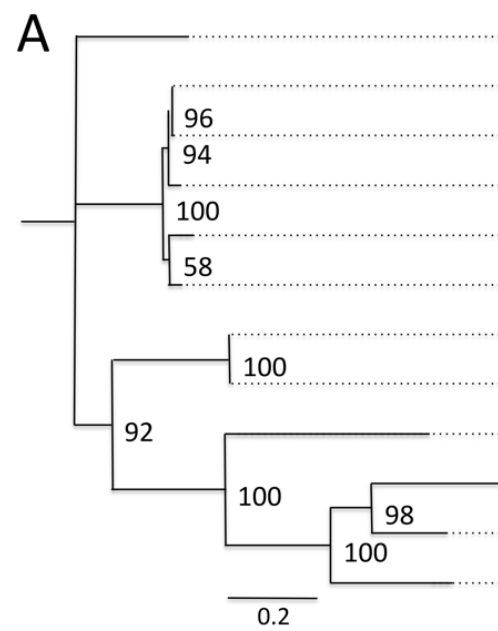

D. ananassae

D. simulans

D. sechellia

D. melanogaster

D. yakuba

D. erecta

D. pseudoobscura

D. persimilis

D. willistoni

D. mojavensis

D. virilis

D. grimshawi

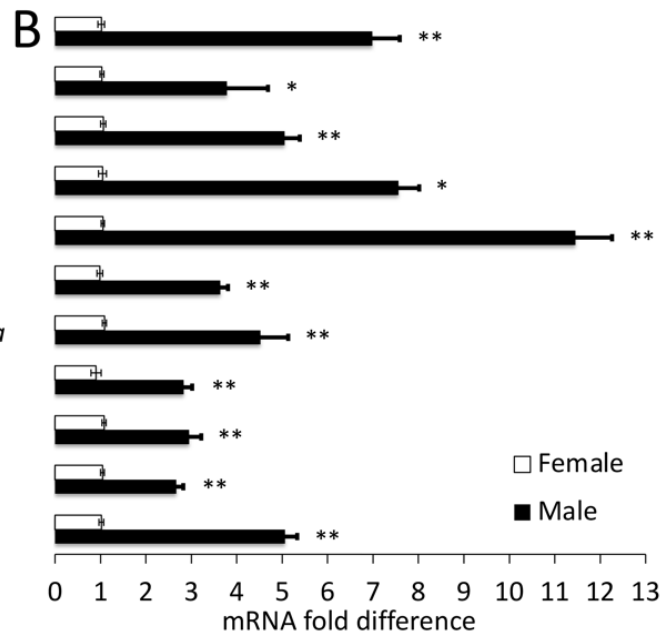

C

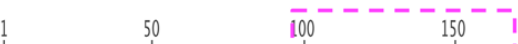

$200 \quad 250$ 300 350

Consensus पா

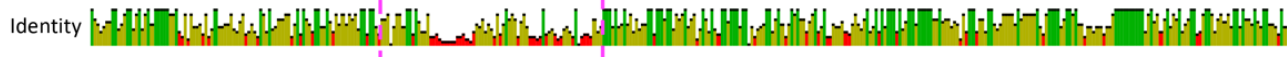

D. ananassae

D. simulans

D. sechelia

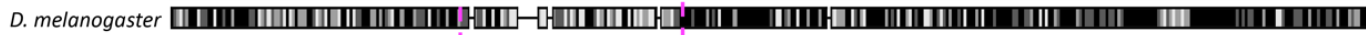

D. yakuba

D. erecta

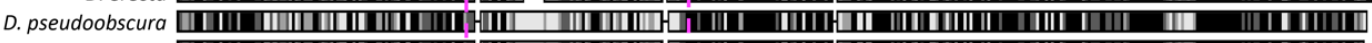

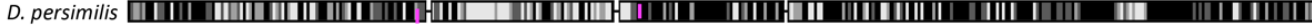

D. willistoni पIIII |س

D. mojavensis IIIII || |س

D. virilis पIIII ||

D. grimshawi

D

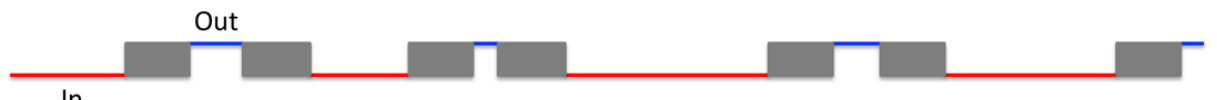

$\mathrm{E}$

In

\section{D. simulans}

D. erecta

D. pseudoobscura

D. persimilis

D. mojavensis

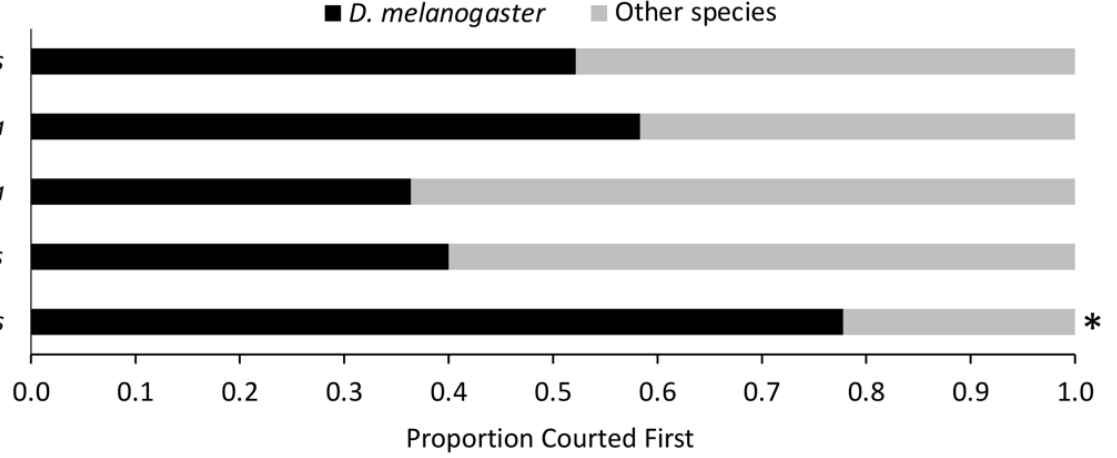

$\mathrm{F}$

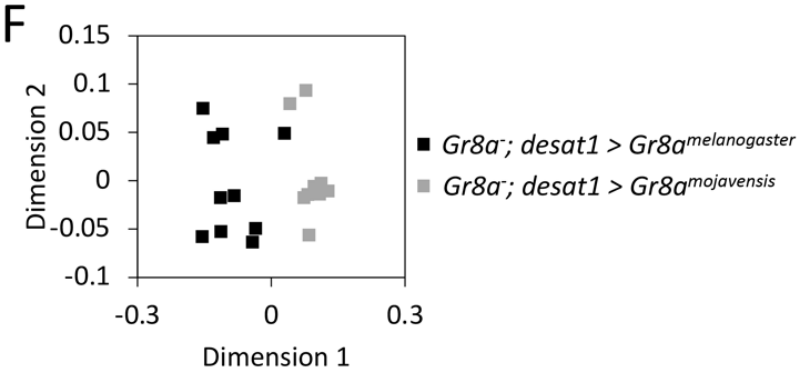

Dimension 1 
Figure 5. Sexually dimorphic Gr8a expression across the Drosophila genus may contribute to species-specific differences in male CHC profiles. (A) Phylogenetic tree of Drosophila Gr8a proteins. Substitution rate $=0.2$. (B) Gr8a mRNA expression is enriched in males relative to females across Drosophila. Black, males; white, females. *, $\mathrm{p}<0.05 ;{ }^{* *}, \mathrm{p}<0.01$; Mann Whitney Rank Sum Test, $\mathrm{n}=4$ /group. Live $D$. grimshawi was not analyzed because live specimens were not available at the Drosophila Species Stock Center (DSSC). (C) Multiple aligned amino acid sequences of Gr8a protein sequences from 12 species across Drosophila. The magenta dashed box highlights a putative hypervariable protein domain. Numbers on top of alignment indicate amino acid number. Black, 100\% identical; Dark Gray, $80-100 \%$ similar; Light Gray, $60-80 \%$ similar; White, less than $60 \%$ similar (Blosum62 score matrix, threshold=1). Bars below consensus represent overall level of amino acid conservation. (D) Gr8a protein topology. Boxes, transmembrane domains; Red lines, intracellular domain; Blue lines, extracellular domains. (E) In female choice assays, D. melanogaster males court females from most other Drosophila species first at an equal proportion as $D$. melanogaster females, but court $D$. mojavensis females first at a lower proportion than $D$. melanogaster females. Assays performed under red light. *, $p<0.05$, Pearson's Chisquared test. (F) Gr8a mutant $D$. melanogaster males with oenocyte-specific $D$. melanogaster Gr8a rescue differ in CHC profile from Gr8a mutant $D$. melanogaster males with oenocyte-specific $D$. mojavensis Gr8a rescue. Depicted as NMDS plot with Bray-Curtis dissimilarity; Gr8a; desat1 > Gr8amelanogaster, D. melanogaster Gr8a oenocyte rescue; Gr8a; desat1 > Gr8a mojavensis,$D$. mojavensis Gr8a oenocyte rescue. Bold letters in legend denote statistical significance, $p<0.05$, permutation MANOVA. 


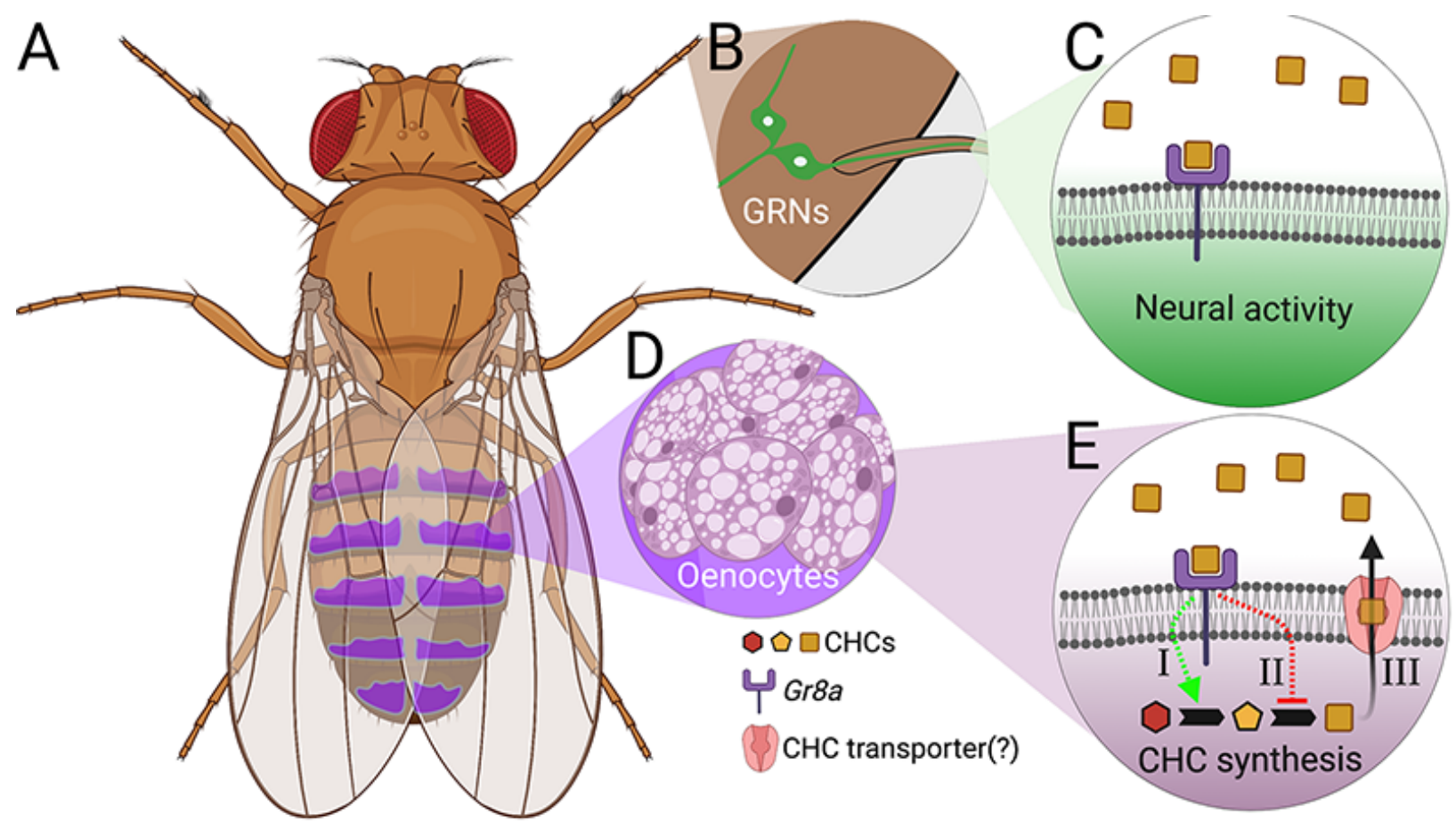

492 Figure 6. Model for the pleiotropic action of Gr8a in the perception and production of pheromones. (A) Drosophila male. The location of $\mathrm{CHC}$-producing oenocytes is shown in magenta. (B) Gr8a-expressing GRNs are located at the last tarsal segment of the prothoracic legs. (C) Gr8a functions as an inhibitory pheromone receptor in a specific subset of leg GRNs. (D) Oenocytes are the primary CHCproducing cells in the male abdomen. (E) Gr8a functions as an autoreceptor in oenocytes, which regulates $\mathrm{CHC}$ synthesis [I-II] and/or $\mathrm{CHC}$ secretion [III] via signaling 499 feedback loops. 
TABLES

\begin{tabular}{|l|l|l|}
\hline Gene & Male & Female \\
\hline Gr2a & - & + \\
\hline Gr8a & + & - \\
\hline Gr10a & + & + \\
\hline Gr21a & - & + \\
\hline Gr22a & + & - \\
\hline Gr22e & + & + \\
\hline Gr36c & + & - \\
\hline Gr58c & + & + \\
\hline Gr59a & + & + \\
\hline Gr59b & + & + \\
\hline Gr63a & + & - \\
\hline Gr64a & + & - \\
\hline Gr64b & + & + \\
\hline Gr64c & + & + \\
\hline Gr64d & + & - \\
\hline Gr66a & + & + \\
\hline Gr89a & + & + \\
\hline Gr93a & - & + \\
\hline Gr93d & + & + \\
\hline Gr97a & + & + \\
\hline Gr98a & + & + \\
\hline Gr98b & - & + \\
\hline Gr98c & + & + \\
\hline Gr98d & + & + \\
\hline Table1 Candidate Grgenes \\
\hline
\end{tabular}

502 Table 1. Candidate Gr genes expressed in male and/or female abdomens. Plus and

503 minus signs indicate whether RT-PCR products were detected. Only genes with positive 504 PCR products in at least one sex are shown. 


\begin{tabular}{|l|l|l|l|l|}
\hline R.T. & Compound & wt \% total & $\begin{array}{l}\text { Gr8a } \% \\
\text { total }\end{array}$ & p value \\
\hline 12.31 & $\mathrm{C}_{21}$ & 0.589 & 1.102 & $<0.001$ \\
\hline 13.24 & Unknown & 0.071 & 0.191 & $<0.001$ \\
\hline 14.2 & $\mathrm{C}_{22}$ & 0.893 & 0.949 & 0.339 \\
\hline 14.34 & $7-\mathrm{C}_{22}$ & 0.362 & 0.490 & $<0.001$ \\
\hline 15.25 & Unknown & 0.128 & 0.226 & $<0.001$ \\
\hline 16.22 & $\mathrm{C}_{23} \& 9-\mathrm{C}_{23}$ & 13.682 & 12.671 & $<0.001$ \\
\hline 16.4 & $7-\mathrm{C}_{23}$ & 30.201 & 35.478 & 0.002 \\
\hline 16.53 & $5-\mathrm{C}_{23}$ & 2.772 & 2.389 & $<0.001$ \\
\hline 16.71 & $\mathrm{C}_{2 \mathrm{~A}}$ & 10.240 & 15.700 & $<0.001$ \\
\hline 18.03 & $\mathrm{C}_{24} \& 9-\mathrm{C}_{24}$ & 0.578 & 0.367 & $<0.001$ \\
\hline 18.19 & $8-\mathrm{C}_{24}$ & 0.742 & 0.493 & $<0.001$ \\
\hline 18.27 & $7-\mathrm{C}_{24}$ & 0.579 & 0.402 & $<0.001$ \\
\hline 18.37 & $6-\mathrm{C}_{24}$ & 0.395 & 0.233 & $<0.001$ \\
\hline 18.46 & $5-\mathrm{C}_{24}$ & 0.040 & 0.047 & 0.943 \\
\hline 19.09 & $2 \mathrm{Me}-\mathrm{C}_{24}$ & 2.426 & 3.240 & $<0.001$ \\
\hline 19.95 & $\mathrm{C}_{25}$ & 0.000 & 2.038 & 0.001 \\
\hline 20.02 & $\mathrm{C}_{25} \& 9-\mathrm{C}_{25}$ & 6.195 & 1.793 & $<0.001$ \\
\hline 20.18 & $7-\mathrm{C}_{25}$ & 14.781 & 4.344 & $<0.001$ \\
\hline 20.42 & $5-\mathrm{C}_{25}$ & 0.296 & 0.000 & $<0.001$ \\
\hline 22.89 & $2 \mathrm{Me}-\mathrm{C}_{26}$ & 6.007 & 6.933 & 0.002 \\
\hline 23.7 & $\mathrm{C}_{27}$ & 1.127 & 0.719 & 0.052 \\
\hline 23.94 & $7-\mathrm{C}_{27}$ & 0.308 & 0.083 & $<0.001$ \\
\hline 26.46 & $2 \mathrm{Me}-\mathrm{C}_{28}$ & 4.992 & 5.775 & 0.078 \\
\hline 27.25 & $\mathrm{C}_{29}$ & 0.351 & 0.317 & 0.574 \\
\hline 29.89 & $2 \mathrm{Me}-\mathrm{C}_{30}$ & 1.245 & 2.353 & $<0.001$ \\
\hline
\end{tabular}

505 Table 2. Male CHCs. Retention time (R.T.), compound, percent total (\% total), and p506 value (Student's t-test or Mann Whitney Rank Sum Test) of each compound as part of 507 the total pheromonal bouquet for wild-type (wt) and Gr8a mutant (Gr8a-) males. 


\begin{tabular}{|l|l|l|l|}
\hline Gene & wt mRNA fold difference & Gr8a- mRNA fold difference & p value \\
\hline desat1 & 1.282 & 0.931 & 0.037 \\
\hline desat2 & 1.413 & 1.270 & 0.506 \\
\hline CG8630 & 0.951 & 1.429 & 0.012 \\
\hline CG9747 & 0.838 & 0.525 & 0.343 \\
\hline CG9743 & 1.060 & 0.959 & 0.373 \\
\hline CG15331 & 0.774 & 1.000 & 0.21 \\
\hline
\end{tabular}

508 Table 3. Desaturase gene expression. Relative mRNA expression of each desaturase 509 gene for wild-type (wt) and Gr8a mutant ( $\left.\mathrm{Gr}^{-} \mathrm{a}\right)$ males. Statistics via Student's t-test. 


\begin{tabular}{|c|c|c|c|c|}
\hline R.T. & Compound & WT \% total & Gr8a \% total & $p$ value \\
\hline 12.31 & $\mathrm{C}_{21}$ & 0.234 & 0.242 & 0.712 \\
\hline 14.2 & $\mathrm{C}_{22}$ & 0.330 & 0.352 & 0.512 \\
\hline 14.34 & $7-C_{22}$ & 0.029 & 0.029 & 0.958 \\
\hline 15.25 & Unknown & 0.238 & 0.200 & 0.128 \\
\hline 16.09 & $\mathrm{C}_{23}$ & 6.481 & 6.580 & 0.748 \\
\hline 16.4 & $7-C_{23}$ & 2.454 & 2.729 & 0.149 \\
\hline 16.39 & $7,11-C_{23}$ & 0.473 & 0.426 & 0.208 \\
\hline 16.53 & $5-C_{23}$ & 0.254 & 0.235 & 0.416 \\
\hline 16.71 & CvA & 1.349 & 1.135 & 0.233 \\
\hline 17.99 & $\mathrm{C}_{24}$ & 0.660 & 0.732 & 0.209 \\
\hline 18.19 & $8-C_{24}$ & 0.156 & 0.174 & 0.334 \\
\hline 19.09 & $2 \mathrm{Me}-\mathrm{C}_{24}$ & 1.214 & 1.104 & 0.155 \\
\hline 19.95 & $\mathrm{C}_{25}$ & 5.404 & 5.683 & 0.336 \\
\hline 20.02 & $\mathrm{C}_{25} \& 9-\mathrm{C}_{25}$ & 3.141 & 3.851 & 0.699 \\
\hline 20.18 & $7-C_{25}$ & 3.144 & 2.999 & 0.422 \\
\hline 20.25 & $7,11-C_{25}$ & 1.822 & 1.684 & 0.315 \\
\hline 20.42 & $5-C_{25}$ & 0.526 & 0.555 & 0.375 \\
\hline 20.47 & $5,9-C_{25}$ & 0.686 & 0.704 & 0.589 \\
\hline 22.89 & $2 \mathrm{Me}-\mathrm{C}_{26}$ & 10.010 & 9.247 & 0.1554 \\
\hline 23.7 & $\mathrm{C}_{27}$ & 3.616 & 3.393 & 0.24 \\
\hline 23.8 & $9-C_{27}$ & 2.498 & 2.739 & 0.937 \\
\hline 23.94 & $7-C_{27}$ & 3.819 & 4.885 & 0.24 \\
\hline 24.1 & $7,11-C_{27}$ & 21.464 & 19.770 & 0.18 \\
\hline 24.28 & $5,9-C_{27}$ & 2.557 & 2.438 & 0.12 \\
\hline 25.85 & $7,11-C_{28}$ & 0.658 & 0.645 & 0.573 \\
\hline 26.46 & $2 \mathrm{Me}-\mathrm{C}_{28}$ & 4.779 & 5.022 & 0.306 \\
\hline 27.25 & $\mathrm{C}_{29}$ & 0.577 & 0.693 & 0.01 \\
\hline 27.7 & $7,11-C_{29}$ & 19.411 & 19.589 & 0.824 \\
\hline 29.89 & $2 \mathrm{Me}-\mathrm{C}_{30}$ & 1.359 & 1.465 & 0.17 \\
\hline 31.03 & $7,11-C_{31}$ & 0.562 & 0.606 & 1 \\
\hline
\end{tabular}


510 Table 4. Mated-female CHCs. Retention time (R.T.), compound, percent total (\% total), 511 and p-value (Student's t-test or Mann Whitney Rank Sum Test) of each compound as 512 part of the total pheromonal bouquet for females mated with wild-type (wt) or Gr8a 513 mutant $\left(\mathrm{Gr} 8 \mathrm{a}^{-}\right)$males. 


\section{METHODS}

515 Animals. Flies were maintained on a standard cornmeal medium under a 12:12 lightdark cycle at 25 Celsius. Unless specifically stated, the D. melanogaster Canton-S (CS) strain served as wild-type control animals. UAS-TNT-E, UAS-TNT-IMP-V1-A, UASmCD8::GFP, UAS-myr::GFP, UAS-Red Stinger, Df(1)BSC663, Df(1)BSC754, Gr8aGAL4, Gr8a ${ }^{1}$, desat1-Gal4, r4-Gal4 and fruP1-LexA fly lines were from the Bloomington Stock center. Originally in the $w^{1118}$ background, the Gr8a ${ }^{1}$ null allele was outcrossed for six generations into the CS wild-type background, which was used as a control. Likewise, the desat1-Gal4 allele was outcrossed for six generations into this Gr8a null background. PromE(800)-GAL4 and PromE(800)>Luciferase were from Joel Levine (The University of Toronto, Canada). The following Drosophila species were obtained from the San Diego Stock Center: D. simulans 14011-0251.192, D. sechellia 140210248.03, D. yakuba 14021-0261.01, D. erecta 14021-0224.00, D. ananassae 140240371.16, D. pseudoobscura 14011-0121.104, D. persimilis 14011-0111.50, D. willistoni 14030-0811.35, D. mojavensis 15081-1352.23, and D. virilis 15010-1051.118. The UAS-Gr8a transgenic lines were generated by cloning the $D$. melanogaster and $D$. mojavensis Gr8a cDNAs into pUAST-attB vector by using 5' EcoRI and 3' Notl restriction sites, followed by $\Phi$ C31 integrase-dependent transgenesis at a Chromosome 2 attP landing site (2L:1476459), as previously described (Zheng et al., 2014). Subsequently, both UAS-Gr8aCDNA lines were transgressed into the $\mathrm{Gr}^{\mathrm{C}} \mathrm{a}^{1}$ background, resulting in complete substitution of the endogenous Gr8a with expression of a Gr8a ortholog. The ppk23-LexA line was generated by integrating our previously described ppk23 promotor DNA fragment (Lu et al., 2012) into the pBPnlsLexA::p65Uw plasmid (Pfeiffer et al., 2010), followed by $Ф C 31$ integrase-dependent transgenesis as above.

The GFP-tagged allele of Gr8a was generated via CRISPR/Cas9-dependent editing using a modified "scarless" strategy by using the sgRNA CGAGCAAGGCGGGAACGATT and a 3XP3>dsRed in the donor plasmid as a reporter for edited animals as previously described (Hill et al., 2017; Hill et al., 2019). Control lines with matching genetic backgrounds were established by selecting DsRed-negative injected animals. The final tagged Gr8a allele was generated by removing the DsRed cassette via the introduction of the piggyBac transposase (Hill et al., 2019).

Immunohistochemistry. To visualize the expression pattern of Gr8a in males and females, Gr8a-GAL4 flies (Lee et al., 2012) were crossed to UAS-CD8::EGFP and liveimaged at 5 days old using a Nikon-A1 confocal microscope. To demonstrate Gr8a expression in oenocytes, abdomens from Gr8a-GAL4NAS-myr::GFP; PromE(800)>Luciferase flies were dissected and immunostained as previously described (Lu et al., 2012; Zheng et al., 2014) by using a Rabbit anti-GFP (1:1000; A11122, Thermo Fisher Scientific) and a mouse anti-luciferase (1:100; 35-6700, Thermo Fisher Scientific) antibodies followed by AlexaFluor 488 anti-rabbit and AlexaFluor 568 anti-mouse secondary antibodies (Both at 1:1000; Thermo Fisher Scientific). To visualize the GR8A protein, abdomens of control flies and flies with CRISPR/Cas9 generated GFP-tagged GR8A were dissected and immunostained as previously described (Lu et al., 2012; Zheng et al., 2014) using a Rabbit anti-GFP antibody (1:1000; A-11122, Thermo Fisher Scientific) followed by AlexaFluor 488 anti-rabbit secondary antibody (1:1000; Thermo Fisher Scientific). 
mRNA expression. Newly eclosed flies were separated by sex under $\mathrm{CO}_{2}$ and aged for 5605 days on standard cornmeal medium. On day 6 , flies were placed in $a-80^{\circ} \mathrm{C}$ freezer 561 until RNA extraction. To separate body parts, frozen flies were placed in $1.5 \mathrm{ml}$ microcentrifuge tubes, dipped in liquid nitrogen, and then vortexed repeatedly until heads, appendages, and bodies were clearly separated. Total RNA was extracted using the Trizol Reagent (Thermo Fisher Scientific) separately from heads, bodies, and appendages for Gr8a expression and from bodies for desaturase enzyme genes. cDNAs were synthesized using SuperScript II reverse transcriptase (Thermo Fisher Scientific) with $500 \mathrm{ng}$ total RNA in a $20 \mathrm{uL}$ reaction. Real-time quantitative RT-PCR was carried out as previously described with Rp49 as the loading control gene (Hill et al., 2017; Hill et al., 2019; Lu et al., 2012; Lu et al., 2014; Zheng et al., 2014). Primer sequences are described in Supplemental Tables 1-3.

Courtship Behavior Assays. Single-pair assays were performed as we have previously published (Lu et al., 2012, 2014). In short, newly eclosed males were kept individually on standard fly food in plastic vials $(12 \times 75 \mathrm{~mm})$. Newly eclosed virgin females were kept in groups of 10 flies. All behaviors were done with 4-7 day-old animals, which were housed under constant conditions of $25^{\circ} \mathrm{C}$ and a $12 \mathrm{~h}: 12 \mathrm{~h}$ lightdark cycle. Courtship was video recorded for $10 \mathrm{~min}$ for male courtship and 15 min for female mating receptivity. Male courtship latency and index were measured as previously described (Lu et al., 2012; Lu et al., 2014). Female receptivity index was defined as the time from the initiation of male courtship until copulation was observed. Unless otherwise indicated, assays were performed under normal light conditions. Male mate-choice assays were performed in round courtship arenas. Briefly, one $D$. melanogaster virgin female and one interspecific virgin female was decapitated under $\mathrm{CO}_{2}$ and placed in the arena. One virgin male D. melanogaster was then aspirated into the arena and behavior was video recorded for 10 minutes. The first female courted (by male wing extension) was noted. Male mate-choice assays were performed under red light conditions.

587 Perfuming studies. Synthetic compounds were synthesized by J.G.M. Perfuming studies were performed using a modified protocol from (Billeter et al., 2009). In short, 3 $\mathrm{mg}$ of each compound was dissolved in $6 \mathrm{~mL}$ hexane (Sigma-Aldrich \#139386-500ML) and $0.5 \mathrm{~mL}$ was pipetted into individual $2 \mathrm{~mL}$ glass vials fitted with $9 \mathrm{~mm}$ PTFE lined caps (Agilent Crosslab, Santa Clara, CA, USA). The hexane was evaporated under a nitrogen gas flow, such that a residue of the compound was left around the bottom onethird of the vial. Control vials were prepared using hexane without a spiked compound. Vials were kept at $-20^{\circ} \mathrm{C}$ until use. Flies used in these trials were collected as described above, kept in single sex groups and aged for 4 days on standard cornmeal medium at $25^{\circ} \mathrm{C}$. 24 hours before perfuming, 20 flies of one or the other sex were placed in glass vials containing standard cornmeal medium $(12 \times 75 \mathrm{~mm})$. To perfume the flies, these groups of 20 flies were dumped without anesthesia into each $2 \mathrm{~mL}$ vial containing the compound of interest, and were vortexed at medium-low speed for 3 pulses of 20 seconds punctuated by 20 second rest periods. Flies were transferred to new food vials and were allowed to recover for one hour. Perfumed flies were then used in courtship behavior assays as described above and the remaining flies were used in pheromone analyses to verify compound transfer. The genotype of flies that were perfumed differed 
based upon the genotype with the lower amount of each compound as determined in

605 Figure $3(\mathrm{~B}, \mathrm{C}, \mathrm{F})$. In all cases, compound transfer was verified by $\mathrm{CHC}$ extraction and 606 GC/MS (Supplemental Table 4).

607 Phylogenetic analysis. Protein sequences of GR8A orthologs from the 12 sequenced 608 Drosophila reference genomes were aligned by using the ClustalW algorithm in the 609 Omega package (Sievers et al., 2011), followed by ProtTest (v2.4) to determine the best 610 model of protein evolution (Abascal et al., 2005). Subsequently, Akaike and Bayesian 611 information criterion scores were used to select the appropriate substitution matrix. We 612 then used a maximum likelihood approach and rapid bootstrapping within RAxML v 7.2.8 Black Box on the Cipres web portal to make a phylogenetic tree (Miller et al., 2010). Visualizations of the bipartition files were made using FigTree v1.3.1 (http://tree.bio.ed.ac.uk/software/figtree/).

Pheromone Analysis. Virgin flies were collected upon eclosion under a light $\mathrm{CO}^{2}$ anesthesia and kept in single-sex vials in groups of 10 with 6 biological replications for each genotype and sex. Virgin flies were aged for 5 days on standard cornmeal medium at $25^{\circ} \mathrm{C}$. To collect mated flies, both females and males were aged for 3 days before single mating pairs were placed in a standard fly vial with standard cornmeal food for 24 hours. The pair was then separated for 24 hours before collection. Copulation was confirmed by the presence of larvae in the vials of mated females several days later. On the morning of day 5, flies were anesthetized under light $\mathrm{CO}^{2}$ and groups of five flies were placed in individual scintillation vials (VWR 74504-20). To extract CHCs, each group of flies was covered by 100 uL hexane (Sigma-Aldrich \#139386-500ML) containing 50 $\mathrm{g} / \mathrm{mL}$ hexacosane (Sigma-Aldrich \#241687-5G) and was washed for ten minutes. Subsequently, hexane washes were transferred into a new $2 \mathrm{ml}$ glass vial containing a $350 \mathrm{uL}$ insert (Thermo Scientific C4000-LV-1W) and were stored at $-20^{\circ} \mathrm{C}$ until shipment to the Millar laboratory. Analyses of $\mathrm{CHC}$ profiles were done by gas chromatography and mass spectroscopy (GC-MS) in the Millar laboratory at UC Riverside as previously described (Chung et al., 2014). Peak areas were measured, and data was normalized to known quantity of internal standard hexacosane (Sigma-Aldrich \#241687-5G). The relative proportion of each compound in each sample was calculated and used in further statistical analysis.

Statistical Analysis. All statistical analyses were performed in R (v 3.6.2). The following functions were used in the base statistics package: t.test() (t-test), wilcox.test() (Mann-Whitney Rank Sum Test), aov() (ANOVA), TukeyHSD() (Tukey's HSD post hoc test), Kruskal.test() (Kruskal-Wallis test), chisq.test() (Pearson's Chi-squared test). Kruskal-Wallis post hoc was performed using the dunn.test.control function in the PMCMR package (Pohlert, 2014). Qualitative CHC data were analyzed through a permutation MANOVA using the adonis function in the vegan package of $R$ with BrayCurtis dissimilarity measures (Oksanen, 2011). CHC profile data were visualized using non-metric multidimensional scaling (metaMDS) function in the vegan package of $R$ 

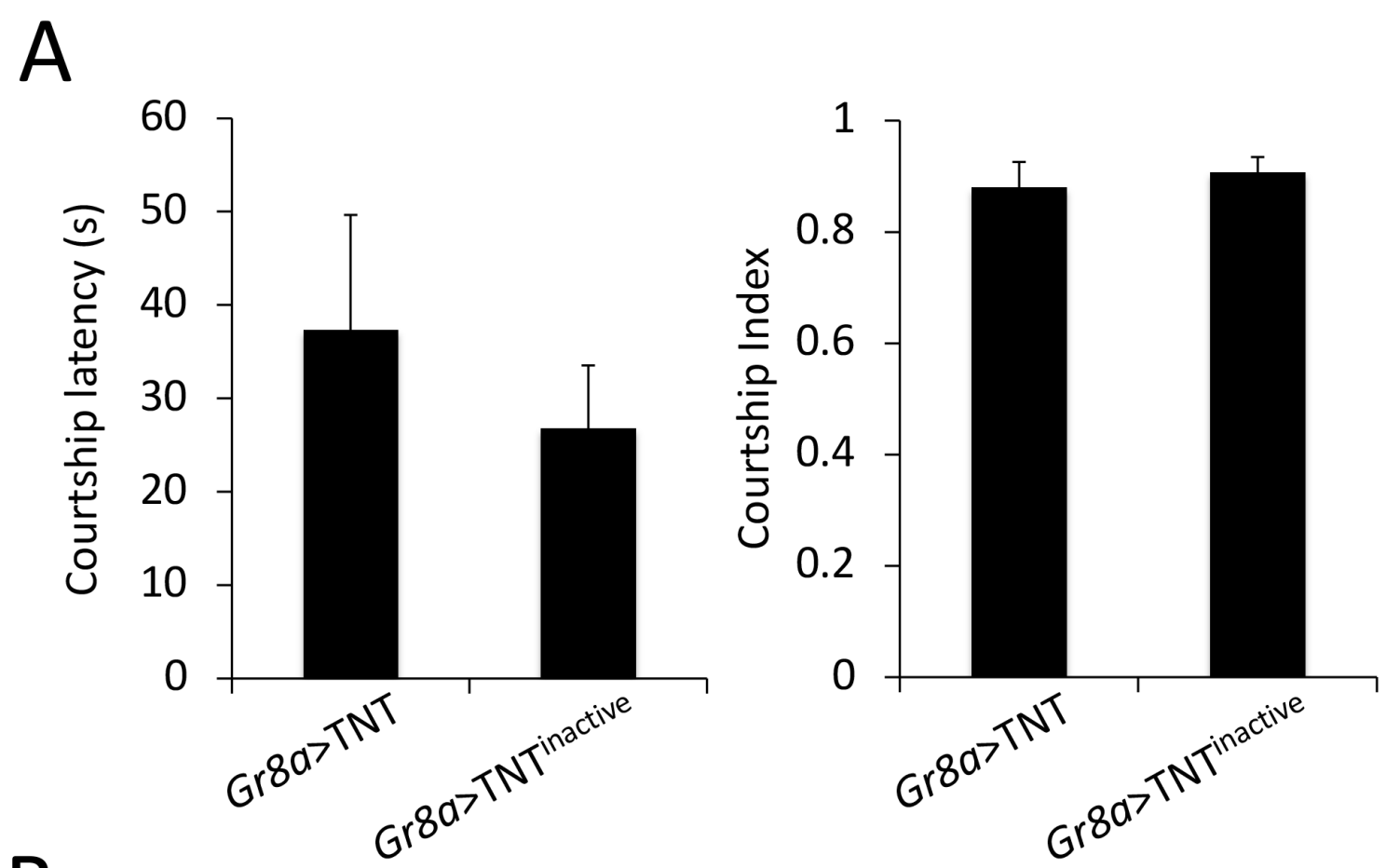

B
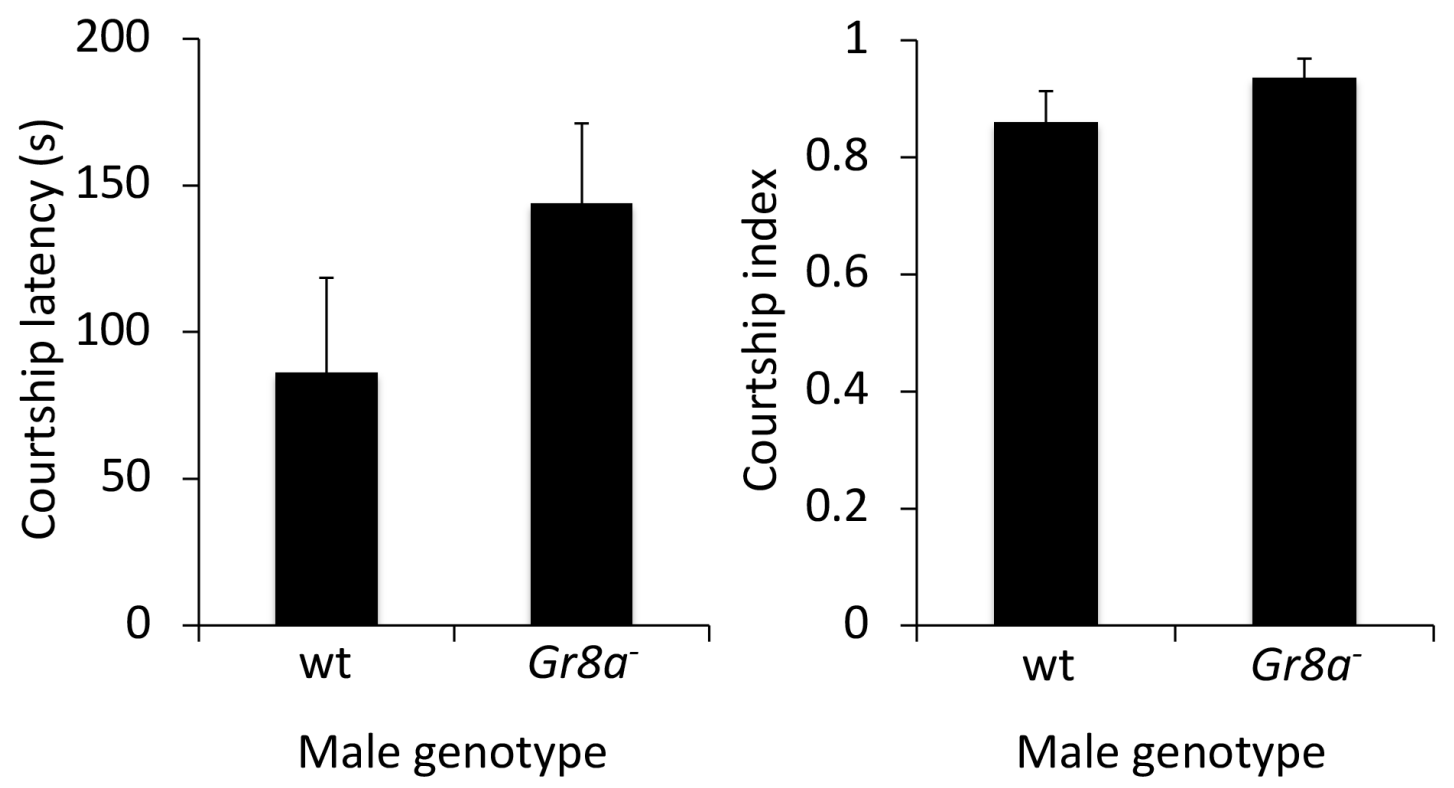

Male genotype

650 Supplemental Figure 1. Gr8a has no effect on male courtship latency or index toward 651 wild-type females. (A) Courtship latency (s) and index of control Gr8a-gal4/UAS-IMP-

652 TNT-V1A (Gr8a>TNT inactive) and Gr8a-gal4/UAS-TNT-E (Gr8a>TNT) mutant males 653 towards wild-type females. (B) Courtship latency (s) and index of wild-type (CS) and 654 Gr8a null (Gr8a-) males toward wild-type decapitated females. Mann Whitney Rank 655 Sum Test, not significant ( $p>0.05)$, $n=15 /$ group. 


\begin{tabular}{|c|c|c|}
\hline Gene & Forward Primer & Reverse Primer \\
\hline Gr10a & AGCGCGAGAGCTTTCGGATGC & AAAGTCCACACAGCATGGGCGG \\
\hline Gr10b & GCGACTGTCCAGAGGATGTGGC & CAGCTGATCAAAGCGAATGCGC \\
\hline Gr21a & AACTACCGGCTCGCCGATCG & AGGTTGCCCCGTGGTCGATGA \\
\hline Gr22a & TGGGACCTTTGGCAGGGAATCG & TGCGATGACTGCAGAAGAGGGT \\
\hline Gr22b & GGTTGTGCATTGCCGCATGCG & CTGCAAAGCCAAGCGAACTCGT \\
\hline Gr22C & GCCGCCAGCGATCTAGCTGAG & CAATGACCGCACAGCAATGCGA \\
\hline Gr22e & TGGTCTTCGTTCAAGCGCTGGT & GCCGGCGATGGCTGCAGAATAAT \\
\hline Gr22f & GATCGCCTGCTGAAGCTGAGTGA & GTGGGTGCACAGCCATGCGAAT \\
\hline Gr23a & AGCGATTTGTGGTGACCGCCA & CATCCGCTGCCTCGCGTTGA \\
\hline Gr28a & TCGCCCTGCCGCACATCAATA & GGGTGAGAGTTCCAAGGCGCT \\
\hline Gr28b & ACTGGAGGCATAGTGCACTCCCT & AGTGGTCAAGGCCCCGCTGAT \\
\hline Gr2a & TTTGCGACCGAACGGCCCAG & GTTGAAGAAGCCAGCGGCGC \\
\hline Gr32a & ACACGGTCACCACCAACTGCT & ACCAAGTATGTTGTGACGGCCGAA \\
\hline Gr33a & ATCGCCACGCACCCCTTACC & GCTCACCACAAAGCAGGCCG \\
\hline Gr36a & GAGGTCGTGTCGTTGCAGCCC & GTGCTCGCTGACGCCATCTGT \\
\hline Gr36b & TGGCATGCAAGGGCTTATGGCT & CCGCAGTTGAGCCACGCGTG \\
\hline Gr36C & CGGCAGAGCAAACATGTTGCACG & ACTGCAAGCCCAGTCCCAGGT \\
\hline Gr39a & TACCCCGAACCGGGACTGGG & CCAGAGCGAAAAATCCATAAGCGGT \\
\hline Gr39b & GGGCGCCAACTGCACATTGG & TCCAATCGAGGGATGGCAGGACA \\
\hline Gr43a & GCTGGCCCTGGCTCCATATGC & AACCCCTTGTTCTTGGCTGCGGC \\
\hline Gr47a & GGTCGGGCCAGAGAAATCGGC & CCCGCTTGGGATGGAGGGCC \\
\hline Gr47b & GCCCTGGGATCCCGAGTACG & TGCCCGATGTCAACTGGTGATCT \\
\hline Gr57a & GGCAAGGAGATGCCTTCGCCT & GCCCATCTTCTGGGTCATGGCG \\
\hline Gr58a & TGTGGATGACGTGACGCGGA & ACGGATGACAACGAAGGTGATCCGA \\
\hline Gr58b & АССТCCTGCAACAACACTGGCC & CCGATTCTGCCTCAGCTGCATGG \\
\hline Gr58C & ACCGCTGTAAGTGGTTGGGACT & TCCAACGATGTGCAGACGTCTCA \\
\hline Gr59a & GCCAAAGTTCTTCGCCCCCGA & TCCAATCGCGTGCTGCTCTCG \\
\hline Gr59b & CAGCCAAGCACTCGTGGAGTGA & TGGCAAACCACATGCTGCGATT \\
\hline Gr59C & CCGAGTGCTGGCGGATCGAAC & CTGGCCAGTTGCAGTTGGAAGT \\
\hline Gr59d & CGTCGATTGCTGGATCAACGCG & TCGACCAAACTCGAAAAGGCCG \\
\hline Gr59e & СCTCTCTGGGAGGAGTCCGTGC & AGGGCAACGCCCATTAGCGACT \\
\hline
\end{tabular}




\begin{tabular}{|c|c|c|}
\hline $\operatorname{Gr} 59 f$ & GCCATGCACGTGGGAAAGGTGT & ACATTGCCCTGAACCGACTTGGA \\
\hline Gr5a & GCCTGCAACGTGCTCGTCCT & TTGAGCGGGACAAGGCGCAG \\
\hline Gr61a & AAAGTGCGGCGCCAGAAGCA & GTGCGACCAGGAGCGAGCAA \\
\hline Gr63a & CGCAAGAAGGGTGACGCGGTG & AAGGGTCCACTCAGGGAGCGC \\
\hline Gr64a & CGCGTGCAATTGGCTGCCTTG & GTCCGCCGCCGTGAGGAATAC \\
\hline Gr64b & GCCCGCGGTCTTTTGGACAGA & CATGGCCAGGACGAGTGAGCG \\
\hline Gr64C & ATGCACTGGCGCATCAGGCA & CCGCCCTCGCAGAAGCTCTT \\
\hline Gr64d & ACCGCGCCTGGATGGTGTTC & TGCACCACGGCACCTTGCAT \\
\hline Gr64e & TGGCCACCACTTGCTCGCTG & ACGCCGCGTGTTAGCCGAAA \\
\hline Gr64f & TCTGGCTAGGCAGTGGCCCC & GCATGGGCCACCGATGGCAT \\
\hline Gr66a & GAGGACCGCAGCCTAAAGGCC & CGCGGTCAACTGGTCGGAGAC \\
\hline Gr77a & CGCAGTTCAGGCCGTCTGCTT & CCACAGGCGCTGACGGCAAATA \\
\hline Gr85a & CTCTCGCCTACATGGCACGC & ATAGTGACGTGCGTGTGCTGC \\
\hline Gr89a & GCTGCATGGAGTTCCCCTGACC & ACATTGCCAGTGGGCGTGAGC \\
\hline Gr8a & ACATACGCATCGCCGTGGACTG & ATGTAGGTGCCCACGGAACAGG \\
\hline Gr92a & TGTCTCCGTTGAGGGAGCGGT & TGGGTGACATCGAATAGGCCCA \\
\hline Gr93a & GGTGAGGCAGTCAGAAGTGCC & CCTCGGACGCCTCAAAGCCAC \\
\hline Gr93b & ACCACGCCATTCTGCGAAGAC & GCCCAAAGGAAACACTCGCAGC \\
\hline Gr93C & TGCGACGCCTGAGTCTGGAGA & CCCAAGGGTCTAACGCGAAACTCG \\
\hline Gr93d & ACTCGGGAACGTGCTCTGGAT & AGAGGCCCAACAAGTTAACCCTGA \\
\hline Gr97a & TGCGTCGCTTCTGCGAGTTAGC & GCGTGCTGCAGATCGAATCGC \\
\hline Gr98a & GTTGAGCACACAAGGGTGGCTG & GCTGTTGCTTGCTGCTGCTCTGA \\
\hline Gr98b & TTGGAGGCTGGAGGGCGATGT & ATGGTGACAAGCAACCCGCCA \\
\hline Gr98C & GCCAGGATTCAGGAGCTGTGCG & CGCTGGTATTTTGTCTGTAGCCGA \\
\hline Gr98d & CTGGCTGGACGCATTTGGGGC & ATGTCAAAGAGGCCACCGCATGT \\
\hline Gr9a & TGTCCTCTGTCTGGGCGACTGG & ACAAGGGACACGAACTGCAGGA \\
\hline
\end{tabular}

656 Supplemental Table 1. Nucleotide sequences for qRT-PCR primers for $D$. 657 melanogaster Grgenes. 


\begin{tabular}{|l|l|l|}
\hline Species & Gr8a Forward Primer & Gr8a Reverse Primer \\
\hline D. melanogaster & TGACCATCAACATACGCATCG & CGTATATGAAGGCGGGAATCTC \\
\hline D. simulans & GAACTTTTCGCTGCAACTCC & ACTTCGGTATAAACTGGATGGTG \\
\hline D. sechellia & GAGATTCCCGCCTTCATATACG & GAGTTGCAGCGAAAAGTTCTG \\
\hline D. erecta & CAGATTCAGAACTTTTCGCTGC & GGTGTAGATCATGTAGGTGCC \\
\hline D. yakuba & TGCCTCGGACTAACAATTCTG & GTGTAGATCATGTAGGTGCCC \\
\hline D. ananassae & AATGTACCGAAGTTTCCAGGG & GCGGGTATGATCAGGAAATAGTC \\
\hline D. pseudoobsCura & CCCGTTTCCGTGACAATATTG & ACCATCTACATATCCGTTGCC \\
\hline D. persimilis & TTTCGCTTCTCCACACTGAC & AGGCGGGCAATATCAAAGAG \\
\hline D. willistoni & GAAATGTTGCCCAGAATAGCC & CCCAAAGCATGTATAACCACTG \\
\hline D. virilis & TCTTCAGATCCAAAACTTTTCGC & TTGGGCATCAGTTGTACGG \\
\hline D. mojavensis & CATATACCCGCCTTTCTCTACAC & GTTCGTGCAGAATTTGTAGCG \\
\hline
\end{tabular}

\begin{tabular}{|l|l|l|}
\hline Species & Rp49 Forward Primer & Rp49 Reverse Primer \\
\hline D. melanogaster & ATCTTGGGCCTGTATGCTG & TGTGATGGGAATTCGTGGG \\
\hline D. simulans & GTCGGATCGATATGCTAAGCTG & CAGATACTGTCCCTTGAAGCG \\
\hline D. sechellia & CATACAGGCCCAAGATCGTG & CAGATACTGTCCCTTGAAGCG \\
\hline D. erecta & GTCGGATCGATATGCTAAGCTG & CAGATACTGTCCCTTGAAGCG \\
\hline D. yakuba & CATACAGGCCCAAGATCGTG & GGCATCAGATACTGTCCCTTG \\
\hline D. ananassae & TACAGGCCCAAGATCGTTAAG & GTACTGACCCTTGAAGCGAC \\
\hline D. pseudoobscura & CCAGCTCCAAAATGACGATTC & TCAATACCCTTAGGCTTGCG \\
\hline D. persimilis & AAGCACTTCATCCGTCACC & TCAATACCCTTAGGCTTGCG \\
\hline D. willistoni & AAGCACTTCATCCGTCACC & GTTGGGCATCAGATATTGGC \\
\hline D. virilis & AGTCGGATCGTTATGCTAAGTTG & TGGAGGGTACGCTTGTTTG \\
\hline D. mojavensis & ACCATTCGTCCAGCATACAG & TTGGCCCTTGAAGCGAC \\
\hline
\end{tabular}

658 Supplemental Table 2. Nucleotide sequences for qRT-PCR primers for $D$.

659 melanogaster Gr8a, Rp49 and orthologs. 


\begin{tabular}{|l|l|l|}
\hline Gene & Forward Primer & Reverse Primer \\
\hline desat1 & TTCTGAACGTAACCTGGCTG & GGGAAGACGTGATGGTAGTTATG \\
\hline desat2 & TTTACTGGAATGAGACGCTGG & CTGGGTAGGATTCATGGTCTTG \\
\hline Fad2 & GTGCCTCAGTCTCAATCTCATC & GTGGTAGTTGTGGTATCCCTC \\
\hline CG8630 & ATGTGATGGCTAAGATCGGAC & TGGTCGGAATGGCACTATTG \\
\hline CG9747 & TCAAGTGGGACAAAGTCATCC & GCCGTAACTCCGAATCCG \\
\hline CG9743 & GGTACTTCTGGAACGAGGATC & TCAGTGGACATGAGGTTCTTG \\
\hline CG15531 & CTCAAGGATGTGGATATGTCGG & ATGGAGGTGGCTAGGGAG \\
\hline rp49 & CACCAAGCACTTCATCCG & TCGATCCGTAACCGATGT \\
\hline
\end{tabular}

660 Supplemental Table 3. Nucleotide sequences for qRT-PCR primers for $D$. 661 melanogaster desaturase enzyme genes. 


\begin{tabular}{|c|c|c|c|c|}
\hline Compound & Genotype & Sex & $\mathrm{ng} / \mathrm{sample}$ & $\mathrm{ng} / \mathrm{fly}$ \\
\hline $\mathrm{C}_{25}$ & wt & male & 1350 & 270 \\
\hline $\mathrm{C}_{25}$ & wt & male & 1170 & 234 \\
\hline $\mathrm{C}_{25}$ & wt & male & 27730 & 5546 \\
\hline $9-C_{25}$ & Gr8a- & male & 67390 & 13478 \\
\hline $9-C_{25}$ & Gr8a- & male & 59380 & 11876 \\
\hline $9-C_{25}$ & Gr8a- & male & 1000 & 200 \\
\hline $7-C_{25}$ & Gr8a- & male & 43790 & 8758 \\
\hline $7-C_{25}$ & Gr8a- & male & 31330 & 6266 \\
\hline $7-C_{25}$ & Gr8a- & male & 24130 & 4826 \\
\hline $5-C_{25}$ & Gr8a- & male & 12050 & 2410 \\
\hline $5-C_{25}$ & Gr8a- & male & 14160 & 2832 \\
\hline $5-C_{25}$ & Gr8a- & male & 1870 & 374 \\
\hline $\mathrm{C}_{27}$ & Gr8a- & male & 890 & 178 \\
\hline $\mathrm{C}_{27}$ & Gr8a- & male & 720 & 144 \\
\hline $\mathrm{C}_{27}$ & Gr8a- & male & 263 & 52.6 \\
\hline $7-C_{27}$ & Gr8a- & male & 36710 & 7342 \\
\hline $7-C_{27}$ & Gr8a- & male & 21250 & 4250 \\
\hline $7-C_{27}$ & Gr8a- & male & 15910 & 3182 \\
\hline $\mathrm{C}_{29}$ & wt & male & 260 & 52 \\
\hline $\mathrm{C}_{29}$ & wt & male & 340 & 68 \\
\hline $\mathrm{C}_{29}$ & wt & male & 890 & 178 \\
\hline $9-C_{25}$ & wt & female & 14060 & 2812 \\
\hline $9-C_{25}$ & wt & female & 13830 & 2766 \\
\hline $7-C_{25}$ & wt & female & 23200 & 4640 \\
\hline $7-C_{25}$ & wt & female & 12780 & 2556 \\
\hline $7-C_{27}$ & wt & female & 1010 & 202 \\
\hline $7-C_{27}$ & wt & female & 1080 & 216 \\
\hline
\end{tabular}

662 Supplemental Table 4. Amount (ng) of each perfumed compound measured in each 663 sample of perfumed flies (5 flies per sample).

664

665

666 
667 Supplemental Data Legends:

668 Figure 1 Data: Average qRT-PCR $\mathrm{C}_{\mathrm{t}}$ scores across 3 technical replicates for each fly 669 sample for Gr8a and rp49 (control).

Figure 2 Data: Copulation latency (s) or courtship index of single-pair courtship trials corresponding to Figure 2.

Figure 3 Data: Amount (ng) of each compound extracted from each sample (5 flies/sample) in Figure 3. Average qRT-PCR $C_{t}$ scores across 3 technical replicates for each fly sample for every desaturase gene measured and rp49 (control). Genes were run on separate $\mathrm{qPCR}$ plates, indicated by plate number in parentheses.

Figure 4 Data: Copulation latency (s) of single-pair courtship trials with perfumed males. Courtship latency (s), courtship index, and copulation latency (s) of single-pair courtship trials with perfumed females.

690

691

692

Figure 5 Data: Average qRT-PCR $C_{t}$ scores across 3 technical replicates for each fly sample for Gr8a and rp49 (control) across Drosophila species. Number of flies courted first by D. melanogaster males in choice assays. Amount (ng) of each compound extracted from each sample (5 flies/sample) in Figure 5.

Figure S1 Data: Courtship latency (s) and index of single-pair courtship trials corresponding to Supplemental Figure 1. 


\section{REFERENCES}

694 Abascal, F., Zardoya, R., and Posada, D. (2005). ProtTest: selection of best-fit models

695 of protein evolution. Bioinformatics 21, 2104-2105. bti263 [pii]

696 10.1093/bioinformatics/bti263.

697 Averhoff, W.W., and Richardson, R.H. (1974). Pheromonal control of mating patterns in

698 Drosophila melanogaster. Behav Genet 4, 207-225.

699 Ben-Shahar, Y. (2015). Editorial overview: Neuroscience: How nervous systems 700 generate behavior: lessons from insects. Current Opinion in Insect Science 12, v-vii. $701 \quad$ 10.1016/j.cois.2015.10.005.

702 Ben-Shahar, Y., Lu, B., Collier, D.M., Snyder, P.M., Schnizler, M., and Welsh, M.J. 703 (2010). The Drosophila gene CheB42a is a novel modifier of Deg/ENaC channel 704 function. PLoS One 5, e9395. 10.1371/journal.pone.0009395.

705 Ben-Shahar, Y., Nannapaneni, K., Casavant, T.L., Scheetz, T.E., and Welsh, M.J. 706 (2007). Eukaryotic operon-like transcription of functionally related genes in Drosophila. 707 Proc Natl Acad Sci U S A 104, 222-227. 10.1073/pnas.0609683104.

708 Benton, R., Vannice, K.S., and Vosshall, L.B. (2007). An essential role for a CD36709 related receptor in pheromone detection in Drosophila. Nature 450, 289-293.

710 10.1038/nature06328.

711 Billeter, J.C., Atallah, J., Krupp, J.J., Millar, J.G., and Levine, J.D. (2009). Specialized 712 cells tag sexual and species identity in Drosophila melanogaster. Nature 461, 987-991. 713 10.1038/nature08495.

714 Billeter, J.C., and Levine, J.D. (2013). Who is he and what is he to you? Recognition in 715 Drosophila melanogaster. Curr Opin Neurobiol 23, 17-23. 10.1016/j.conb.2012.08.009.

716 Blomquist, G.J., and Bagnères, A.-G. (2010). Insect hydrocarbons : biology, 717 biochemistry, and chemical ecology (Cambridge University Press). 
718 Boake, C.R. (1991). Coevolution of senders and receivers of sexual signals: Genetic 719 coupling and genetic correlations. Trends Ecol Evol 6, 225-227. 10.1016/0169720 5347(91)90027-U.

721 Bousquet, F., Nojima, T., Houot, B., Chauvel, I., Chaudy, S., Dupas, S., Yamamoto, D., 722 and Ferveur, J.F. (2012). Expression of a desaturase gene, desat1, in neural and 723 nonneural tissues separately affects perception and emission of sex pheromones in 724 Drosophila. Proc Natl Acad Sci U S A 109, 249-254. 10.1073/pnas.1109166108.

725 Bray, S., and Amrein, H. (2003). A putative Drosophila pheromone receptor expressed 726 in male-specific taste neurons is required for efficient courtship. Neuron 39, 1019-1029.

727 Brooks, R., Hunt, J., Blows, M.W., Smith, M.J., Bussiere, L.F., and Jennions, M.D.

728 (2005). Experimental evidence for multivariate stabilizing sexual selection. Evolution 59, 729 871-880. Doi 10.1111/J.0014-3820.2005.Tb01760.X.

730 Butlin, R.K., and Ritchie, M.G. (1989). Genetic coupling in mate recognition systems:

731 what is the evidence? Biological Journal of the Linnean Society 37, 237-246.

732 10.1111/j.1095-8312.1989.tb01902.x.

733 Butlin, R.K., and Trickett, A.J. (1997). Can Population Genetic Simulations Help to 734 Interpret Pheromone Evolution? In Insect Pheromone Research, R.T. Cardé, and A.K. 735 Minks, eds. (Springer US), pp. 548-562. 10.1007/978-1-4615-6371-6_47.

736 Chebib, J., and Guillaume, F. (2021). Pleiotropy or linkage? Their relative contributions 737 to the genetic correlation of quantitative traits and detection by multitrait GWA studies. 738 Genetics 219. 10.1093/genetics/iyab159.

739 Chenoweth, S.F., and Blows, M.W. (2006). Dissecting the complex genetic basis of 740 mate choice. Nat Rev Genet 7, 681-692. 10.1038/nrg1924.

741 Chung, H., and Carroll, S.B. (2015). Wax, sex and the origin of species: Dual roles of 742 insect cuticular hydrocarbons in adaptation and mating. Bioessays $37,822-830$.

743 10.1002/bies.201500014. 
744 Chung, H., Loehlin, D.W., Dufour, H.D., Vaccarro, K., Millar, J.G., and Carroll, S.B. 745 (2014). A single gene affects both ecological divergence and mate choice in Drosophila. 746 Science 343, 1148-1151. 10.1126/science.1249998.

747 Clowney, E.J., Iguchi, S., Bussell, J.J., Scheer, E., and Ruta, V. (2015). Multimodal 748 Chemosensory Circuits Controlling Male Courtship in Drosophila. Neuron 87, 1036749 1049. 10.1016/j.neuron.2015.07.025.

750 Clyne, P.J., Warr, C.G., and Carlson, J.R. (2000). Candidate Taste Receptors in 751 Drosophila. Science 287, 1830-1834. 10.1126/science.287.5459.1830.

752 Datta, S.R., Vasconcelos, M.L., Ruta, V., Luo, S., Wong, A., Demir, E., Flores, J., 753 Balonze, K., Dickson, B.J., and Axel, R. (2008). The Drosophila pheromone cVA 754 activates a sexually dimorphic neural circuit. Nature 452, 473-477. nature06808 [pii] $75510.1038 /$ nature06808.

756 Dunipace, L., Meister, S., McNealy, C., and Amrein, H. (2001). Spatially restricted 757 expression of candidate taste receptors in the Drosophila gustatory system. Curr Biol 758 11, 822-835. S0960-9822(01)00258-5 [pii].

759 Dweck, H.K., Ebrahim, S.A., Thoma, M., Mohamed, A.A., Keesey, I.W., Trona, F., 760 Lavista-Llanos, S., Svatos, A., Sachse, S., Knaden, M., and Hansson, B.S. (2015). 761 Pheromones mediating copulation and attraction in Drosophila. Proc Natl Acad Sci U S 762 A 112, E2829-2835. 10.1073/pnas.1504527112.

763 Dweck, H.K.M., and Carlson, J.R. (2020). Molecular Logic and Evolution of Bitter Taste 764 in Drosophila. Curr Biol 30, 17-30 e13. 10.1016/j.cub.2019.11.005.

765 Dyer, K.A., White, B.E., Sztepanacz, J.L., Bewick, E.R., and Rundle, H.D. (2014).

766 Reproductive character displacement of epicuticular compounds and their contribution 767 to mate choice in Drosophila subquinaria and Drosophila recens. Evolution 68, 1163768 1175. 10.1111/evo.12335.

769 Ferveur, J.F. (2005). Cuticular hydrocarbons: their evolution and roles in Drosophila 770 pheromonal communication. Behav Genet 35, 279-295. 10.1007/s10519-005-3220-5. 
771 Ford, C.P. (2014). The role of D2-autoreceptors in regulating dopamine neuron activity

772 and transmission. Neuroscience 282, 13-22. 10.1016/j.neuroscience.2014.01.025.

773 Fukamachi, S., Kinoshita, M., Aizawa, K., Oda, S., Meyer, A., and Mitani, H. (2009).

774 Dual control by a single gene of secondary sexual characters and mating preferences in 775 medaka. BMC Biol 7, 64. 10.1186/1741-7007-7-64.

776 Gutierrez, E., Wiggins, D., Fielding, B., and Gould, A.P. (2007). Specialized hepatocyte-

777 like cells regulate Drosophila lipid metabolism. Nature 445, 275-280.

778 10.1038/nature05382.

779 Heinen-Kay, J.L., Nichols, R.E., and Zuk, M. (2020). Sexual signal loss, pleiotropy, and 780 maintenance of a male reproductive polymorphism in crickets. Evolution 74, 1002-1009. 781 10.1111/evo.13952.

782 Hill, A., Zheng, X., Li, X., McKinney, R., Dickman, D., and Ben-Shahar, Y. (2017). The 783 Drosophila Postsynaptic DEG/ENaC Channel ppk29 Contributes to Excitatory 784 Neurotransmission. J Neurosci 37, 3171-3180. 10.1523/JNEUROSCI.3850-16.2017.

785 Hill, A.S., Jain, P., Folan, N.E., and Ben-Shahar, Y. (2019). The Drosophila ERG 786 channel seizure plays a role in the neuronal homeostatic stress response. PLoS Genet 787 15, e1008288. 10.1371/journal.pgen.1008288.

788 Howard, R.W., and Blomquist, G.J. (2005). ECOLOGICAL, BEHAVIORAL, AND 789 BIOCHEMICAL ASPECTS OF INSECT HYDROCARBONS. Annual Review of 790 Entomology 50, 371-393. 10.1146/annurev.ento.50.071803.130359.

791 Hoy, R.R., Hahn, J., and Paul, R.C. (1977). Hybrid cricket auditory behavior: evidence 792 for genetic coupling in animal communication. Science 195, 82-84.

$79310.1126 /$ science.831260.

794 Jin, X., Ha, T.S., and Smith, D.P. (2008). SNMP is a signaling component required for 795 pheromone sensitivity in Drosophila. Proc Natl Acad Sci U S A 105, 10996-11001.

7960803309105 [pii]

$797 \quad 10.1073 /$ pnas.0803309105. 
798 Kallman, B.R., Kim, H., and Scott, K. (2015). Excitation and inhibition onto central

799 courtship neurons biases Drosophila mate choice. eLife 4. 10.7554/elife.11188.

800 Khallaf, M.A., Cui, R., Weissflog, J., Erdogmus, M., Svatos, A., Dweck, H.K.M., 801 Valenzano, D.R., Hansson, B.S., and Knaden, M. (2021). Large-scale characterization 802 of sex pheromone communication systems in Drosophila. Nat Commun 12, 4165.

803 10.1038/s41467-021-24395-z.

804 Kirkpatrick, M., and Hall, D.W. (2004). Sexual selection and sex linkage. Evolution 58, 805 683-691. 10.1111/j.0014-3820.2004.tb00401.x.

806 Koh, T.W., He, Z., Gorur-Shandilya, S., Menuz, K., Larter, N.K., Stewart, S., and 807 Carlson, J.R. (2014). The Drosophila IR20a clade of ionotropic receptors are candidate 808 taste and pheromone receptors. Neuron 83, 850-865. 10.1016/j.neuron.2014.07.012.

809 Krupp, J.J., Kent, C., Billeter, J.C., Azanchi, R., So, A.K., Schonfeld, J.A., Smith, B.P., 810 Lucas, C., and Levine, J.D. (2008). Social experience modifies pheromone expression 811 and mating behavior in male Drosophila melanogaster. Curr Biol 18, 1373-1383. S0960812 9822(08)01034-8 [pii]

813 10.1016/j.cub.2008.07.089.

814 Kurtovic, A., Widmer, A., and Dickson, B.J. (2007). A single class of olfactory neurons 815 mediates behavioural responses to a Drosophila sex pheromone. Nature 446, 542-546.

816 nature05672 [pii]

$817 \quad 10.1038 /$ nature05672.

818 Labeur, C., Dallerac, R., and Wicker-Thomas, C. (2002). Involvement of desat1 gene in 819 the control of Drosophila melanogaster pheromone biosynthesis. Genetica 114, 269820 274. 10.1023/a:1016223000650.

821 Lacaille, F., Hiroi, M., Twele, R., Inoshita, T., Umemoto, D., Manière, G., Marion-Poll, F., 822 Ozaki, M., Francke, W., Cobb, M., et al. (2007). An Inhibitory Sex Pheromone Tastes 823 Bitter for Drosophila Males. PLoS ONE 2, e661. 10.1371/journal.pone.0000661. 
824 Lande, R. (1980). The Genetic Covariance between Characters Maintained by

825 Pleiotropic Mutations. Genetics 94, 203-215. 10.1093/genetics/94.1.203.

826 Laturney, M., and Billeter, J.C. (2016). Drosophila melanogaster females restore their

827 attractiveness after mating by removing male anti-aphrodisiac pheromones. Nat

828 Commun 7, 12322. 10.1038/ncomms12322.

829 Lebreton, S., Grabe, V., Omondi, A.B., Ignell, R., Becher, P.G., Hansson, B.S., Sachse,

830 S., and Witzgall, P. (2014). Love makes smell blind: mating suppresses pheromone 831 attraction in Drosophila females via Or65a olfactory neurons. Scientific reports 4, 7119.

$83210.1038 /$ srep07119.

833 Lee, Y., Kang, M.J., Shim, J., Cheong, C.U., Moon, S.J., and Montell, C. (2012).

834 Gustatory receptors required for avoiding the insecticide L-canavanine. J Neurosci 32,

835 1429-1435. 10.1523/JNEUROSCI.4630-11.2012.

836 Lee, Y., Moon, S.J., and Montell, C. (2009). Multiple gustatory receptors required for the 837 caffeine response in Drosophila. Proc Natl Acad Sci U S A 106, 4495-4500.

8380811744106 [pii]

839 10.1073/pnas.0811744106.

840 Leitner, N., and Ben-Shahar, Y. (2020). The neurogenetics of sexually dimorphic 841 behaviors from a postdevelopmental perspective. Genes Brain Behav 19, e12623. $842 \quad 10.1111 / \mathrm{gbb} .12623$.

843 Lu, B., LaMora, A., Sun, Y., Welsh, M.J., and Ben-Shahar, Y. (2012). ppk23-Dependent 844 chemosensory functions contribute to courtship behavior in Drosophila melanogaster. 845 PLoS Genet 8, e1002587. 10.1371/journal.pgen.1002587.

846 Lu, B., Zelle, K.M., Seltzer, R., Hefetz, A., and Ben-Shahar, Y. (2014). Feminization of 847 pheromone-sensing neurons affects mating decisions in Drosophila males. Biology 848 open 3, 152-160. 10.1242/bio.20147369. 
849 McKinney, R.M., Vernier, C., and Ben-Shahar, Y. (2015). The neural basis for insect 850 pheromonal communication. Curr Opin Insect Sci 12, 86-92.

$851 \quad$ 10.1016/j.cois.2015.09.010.

852 Miller, M.A., Pfeiffer, W., and Schwartz, T. (2010). Creating the CIPRES Science 853 Gateway for inference of large phylogenetic trees. 14-14 Nov. 2010. pp. 1-8.

854 Miyamoto, T., and Amrein, H. (2008). Suppression of male courtship by a Drosophila 855 pheromone receptor. Nat Neurosci 11, 874-876. nn.2161 [pii]

$85610.1038 / \mathrm{nn} .2161$.

857 Moon, S.J., Lee, Y., Jiao, Y., and Montell, C. (2009). A Drosophila gustatory receptor 858 essential for aversive taste and inhibiting male-to-male courtship. Curr Biol 19, $1623-$ 859 1627. S0960-9822(09)01487-0 [pii]

$860 \quad 10.1016 /$ j.cub.2009.07.061.

861 Oksanen, J. (2011). Multivariate analysis of ecological communities in R: vegan tutorial. 862 R package version 1, 1-43.

863 Oksanen, J. (2015). Vegan: an introduction to ordination. URL http://cran. r-project. $864 \mathrm{org} / \mathrm{web} /$ packages/vegan/vignettes/introvegan. pdf 8, 19.

865 Pfeiffer, B.D., Ngo, T.T., Hibbard, K.L., Murphy, C., Jenett, A., Truman, J.W., and Rubin, 866 G.M. (2010). Refinement of tools for targeted gene expression in Drosophila. Genetics 867 186, 735-755. 10.1534/genetics.110.119917.

868 Pikielny, C.W. (2012). Sexy DEG/ENaC channels involved in gustatory detection of fruit 869 fly pheromones. Sci Signal 5, pe48. 10.1126/scisignal.2003555.

870 Pohlert, T. (2014). The pairwise multiple comparison of mean ranks package (PMCMR).

871 Poudel, S., Kim, Y., Kim, Y.T., and Lee, Y. (2015). Gustatory receptors required for 872 sensing umbelliferone in Drosophila melanogaster. Insect Biochem Mol Biol 66, 110873 118. 10.1016/j.ibmb.2015.10.010. 
874 Scott, K., Brady, R., Jr., Cravchik, A., Morozov, P., Rzhetsky, A., Zuker, C., and Axel, R.

875 (2001). A chemosensory gene family encoding candidate gustatory and olfactory

876 receptors in Drosophila. Cell 104, 661-673. S0092-8674(01)00263-X [pii].

877 Shahandeh, M.P., Pischedda, A., and Turner, T.L. (2018). Male mate choice via

878 cuticular hydrocarbon pheromones drives reproductive isolation between Drosophila

879 species. Evolution 72, 123-135. 10.1111/evo.13389.

880 Shankar, S., Chua, J.Y., Tan, K.J., Calvert, M.E.K., Weng, R., Ng, W.C., Mori, K., and 881 Yew, J.Y. (2015). The neuropeptide tachykinin is essential for pheromone detection in a 882 gustatory neural circuit. eLife 4. 10.7554/elife.06914.

883 Shaw, K.L., Ellison, C.K., Oh, K.P., and Wiley, C. (2011). Pleiotropy, "sexy" traits, and 884 speciation. Behavioral Ecology 22, 1154-1155.

885 Shaw, K.L., and Lesnick, S.C. (2009). Genomic linkage of male song and female 886 acoustic preference QTL underlying a rapid species radiation. Proc Natl Acad Sci U S A 887 106, 9737-9742. 10.1073/pnas.0900229106.

888 Shim, J., Lee, Y., Jeong, Y.T., Kim, Y., Lee, M.G., Montell, C., and Moon, S.J. (2015).

889 The full repertoire of Drosophila gustatory receptors for detecting an aversive 890 compound. Nat Commun 6, 8867. 10.1038/ncomms9867.

891 Shirangi, T.R., Dufour, H.D., Williams, T.M., and Carroll, S.B. (2009). Rapid evolution of 892 sex pheromone-producing enzyme expression in Drosophila. PLoS Biol 7, e1000168.

893 10.1371/journal.pbio.1000168.

894 Sievers, F., Wilm, A., Dineen, D., Gibson, T.J., Karplus, K., Li, W., Lopez, R., 895 McWilliam, H., Remmert, M., Söding, J., et al. (2011). Fast, scalable generation of high896 quality protein multiple sequence alignments using Clustal Omega. Molecular Systems 897 Biology 7.

898 Singh, N.D., and Shaw, K.L. (2012). On the scent of pleiotropy. Proc Natl Acad Sci U S 899 A 109, 5-6. 10.1073/pnas.1118531109. 
900 Stagkourakis, S., Kim, H., Lyons, D.J., and Broberger, C. (2016). Dopamine

901 Autoreceptor Regulation of a Hypothalamic Dopaminergic Network. Cell reports.

902 10.1016/j.celrep.2016.03.062.

903 Steiger, S., Schmitt, T., and Schaefer, H.M. (2011). The origin and dynamic evolution of 904 chemical information transfer. Proc Biol Sci 278, 970-979. 10.1098/rspb.2010.2285.

905 Sung, H.Y., Jeong, Y.T., Lim, J.Y., Kim, H., Oh, S.M., Hwang, S.W., Kwon, J.Y., and 906 Moon, S.J. (2017). Heterogeneity in the Drosophila gustatory receptor complexes that 907 detect aversive compounds. Nat Commun 8, 1484. 10.1038/s41467-017-01639-5.

908 Sweigart, A.L. (2010). The genetics of postmating, prezygotic reproductive isolation 909 between Drosophila virilis and D. americana. Genetics 184, 401-410.

$910 \quad 10.1534 /$ genetics.109.111245.

911 Symonds, M.R., and Elgar, M.A. (2008). The evolution of pheromone diversity. Trends 912 Ecol Evol 23, 220-228. 10.1016/j.tree.2007.11.009.

913 Symonds, M.R., and Wertheim, B. (2005). The mode of evolution of aggregation 914 pheromones in Drosophila species. J Evol Biol 18, 1253-1263. 10.1111/j.1420915 9101.2005.00971.x.

916 Thistle, R., Cameron, P., Ghorayshi, A., Dennison, L., and Scott, K. (2012). Contact 917 Chemoreceptors Mediate Male-Male Repulsion and Male-Female Attraction during 918 Drosophila Courtship. Cell 149, 1140-1151. S0092-8674(12)00523-5 [pii] 919 10.1016/j.cell.2012.03.045.

920 Toda, H., Zhao, X., and Dickson, B.J. (2012). The Drosophila Female Aphrodisiac 921 Pheromone Activates ppk23+ Sensory Neurons to Elicit Male Courtship Behavior. Cell 922 reports doi:10.1016/j.celrep.2012.05.007, 599-607. 10.1016/j.celrep.2012.05.007.

923 van der Goes van Naters, W., and Carlson, J.R. (2007). Receptors and neurons for fly 924 odors in Drosophila. Curr Biol 17, 606-612. S0960-9822(07)01020-2 [pii]

925 10.1016/j.cub.2007.02.043. 
926 Vijayan, V., Thistle, R., Liu, T., Starostina, E., and Pikielny, C.W. (2014). Drosophila 927 pheromone-sensing neurons expressing the ppk25 ion channel subunit stimulate male 928 courtship and female receptivity. PLoS Genet 10, e1004238.

929 10.1371/journal.pgen.1004238.

930 Wang, Z., Singhvi, A., Kong, P., and Scott, K. (2004). Taste representations in the 931 Drosophila brain. Cell 117, 981-991. 10.1016/j.cell.2004.06.011

932 S009286740400577X [pii].

933 Watanabe, K., Toba, G., Koganezawa, M., and Yamamoto, D. (2011). Gr39a, a highly 934 diversified gustatory receptor in Drosophila, has a role in sexual behavior. Behav Genet 935 41, 746-753. 10.1007/s10519-011-9461-6.

936 Wiley, C., Ellison, C.K., and Shaw, K.L. (2012). Widespread genetic linkage of mating 937 signals and preferences in the Hawaiian cricket Laupala. Proc Biol Sci 279, 1203-1209. 938 10.1098/rspb.2011.1740.

939 Wyatt, T.D. (2014). Pheromones and animal behavior : chemical signals and signatures, 940 Second edition. Edition (Cambridge University Press).

941 Yang, C.-h., Rumpf, S., Xiang, Y., Gordon, M.D., Song, W., Jan, L.Y., and Jan, Y.-N. 942 (2009). Control of the Postmating Behavioral Switch in Drosophila Females by Internal 943 Sensory Neurons. Neuron 61, 519-526.

944 Yew, J.Y., and Chung, H. (2015). Insect pheromones: An overview of function, form, 945 and discovery. Prog Lipid Res 59, 88-105. 10.1016/j.plipres.2015.06.001.

946 Yew, J.Y., Dreisewerd, K., Luftmann, H., Muthing, J., Pohlentz, G., and Kravitz, E.A. 947 (2009). A new male sex pheromone and novel cuticular cues for chemical 948 communication in Drosophila. Curr Biol 19, 1245-1254. S0960-9822(09)01313-X [pii] 949 10.1016/j.cub.2009.06.037.

950 Zheng, X., Valakh, V., DiAntonio, A., and Ben-Shahar, Y. (2014). Natural antisense 951 transcripts regulate the neuronal stress response and excitability. eLife 3, e01849. 952 10.7554/eLife.01849. 
953 